\title{
Atomistic modelling of TiAl I. Bond-order potentials with environmental dependence
}

\author{
S. Znam $\dagger$, D. Nguyen-Manh $\ddagger \S$, D. G. Pettifor $\ddagger$ and V. Vitek $\dagger$ \\ $\dagger$ Department of Materials Science and Engineering, University of Pennsylvania, \\ Philadelphia, Pennsylvania 19104-6267, USA \\ $\ddagger$ Department of Materials, University of Oxford, Parks Road, Oxford OX1 3PH, \\ UK
}

[Received 11 April 2002 and accepted in revised form 3 October 2002]

\begin{abstract}
Bond-order potentials (BOPs) for $\mathrm{L}_{0}$ TiAl have been developed and constructed within a tight-binding framework. In addition to the usual attractive bond-energy contribution arising from the formation of covalent bonds and pairwise contribution describing the overlap repulsion and electrostatic interaction, we have included an environmentally dependent term to represent the strong repulsion experienced by the valence sp electrons in transition metals and their alloys. The latter contribution is crucial for reproducing the negative Cauchy pressures of TiAl and other transition-metalbased intermetallic compounds. The constructed BOPs have been tested in the following ways: firstly, examination of the mechanical stability of the tetragonal $\mathrm{L1}_{0}$ lattice with respect to large deformations and other crystal structures with the same stoichiometry; secondly, calculation of the $\gamma$ surface for $\{111\}$ planes and related evaluation of the energies of stacking-fault-type defects; thirdly, calculation of energies of the $\gamma-\gamma$ interfaces that are present in the lamellar TiAl and energies associated with the formations of point defects in TiAl. The results of all these calculations show very good agreement with various ab-initio calculations. Importantly, we find that this potential is transferable to the different bonding environment in the hexagonal $\mathrm{D}_{19} \mathrm{Ti}_{3} \mathrm{Al}$. Hence these BOPs are suitable for atomistic study of dislocations and other extended defects not only in $\mathrm{Ll}_{0} \mathrm{TiAl}$ but also in $\mathrm{Ti}_{3} \mathrm{Al}$ and possibly structures with other titanium-rich stoichiometries.
\end{abstract}

\section{$\S 1$. INTRODUCTION}

Current interest in intermetallic compounds stems from their attractive combination of physical and mechanical properties, and in particular exceptionally high strength, hardness, creep and corrosion resistance at high temperatures (Westbrook 1996, Stoloff et al. 2000, Yamaguchi et al. 2000, Westbrook and Fleisher 2002). Common negative characteristics of these materials are their small or even non-existent ductility at ambient temperatures. In this respect, the most promising hightemperature intermetallics are titanium aluminides based on $\gamma$-TiAl with the tetragonal L1 $1_{0}$ structure (Kim 1998, Dimiduk 1998, 1999, Tetsui 1999, Yamaguchi et al. 2000, Loria 2000, 2001). These alloys display dramatically different microstructures

$\S$ Author for correspondence. Email: manh.nguyen@materials.ox.ac.uk. 
for different compositions near the 50:50 aluminium : titanium ratio (McCullough et al. 1988, Froes and Suryanarayama 1996, Zhang et al. 1997). Al-rich alloys have a single-phase tetragonal $\mathrm{L}_{0}$ structure but stoichiometric and Ti-rich alloys exhibit a two-phase lamellar structure, consisting of majority layers of $\mathrm{L}_{0}$ TiAl and minority layers of hexagonal $\mathrm{D}_{19} \mathrm{Ti}_{3} \mathrm{Al}$. Interestingly, it is the two-phase lamellar form that results in the toughest materials at room temperature although its components are quite brittle in single-crystal form (Kim 1994, Cahn 1995, Yamaguchi et al. 1996, Appel and Wagner 1998).

The physical understanding of the mechanical properties of $\mathrm{L}_{0} \mathrm{TiAl}$, in either the single phase or the lamellar form, is still rather incomplete. It is now well established that the main deformation modes in $\gamma$-TiAl are slip and twinning, both of them operating on the close-packed $\{111\}$ planes. The slip occurs via two types of dislocation: ordinary dislocations with Burgers vectors $\frac{1}{2}\langle 110\rangle$ and superdislocations with the Burgers vector $\langle 101\rangle$. The twinning is of the $\{111\}\langle 112\rangle$ type, in a similar way to the underlying fcc structure. However, it is not the low-temperature deformation mode as in many materials but occurs at ambient and high temperatures. Different deformation modes dominate in the single-phase and lamellar TiAl and their activity is strongly dependent on temperature (Inui et al. 1992a, 1995, 1997, Yamaguchi et al. 1996). In single-phase alloys an anomalous increase in the yield stress with increasing temperature was also observed (Kawabata et al. 1985, 1991, Inui et al. 1997). Moreover, in the lamellar phase, the yield stress is a function of the angle of the loading axis with respect to the plane parallel to the lamellar boundaries (Fujiwara et al. 1990) and recent experimental studies suggest that a contribution of ordinary slip and twinning occurs such that it exactly cancels any strain component parallel to the lamellar interfaces (Kishida et al. 1998, 1999, Kim et al. 1999). Understanding of these deformation properties cannot be based on the standard elastic theory of dislocations since the above-mentioned phenomena cannot be conceivably controlled by long-range dislocation interactions.

The ductility of crystalline materials is controlled by the motion of dislocations and it is a common feature of complex structures that dislocations possess nonplanar sessile cores and thus very high Peierls stresses (for reviews see for example Duesbery and Richardson (1991) and Vitek (1992)). This characteristic of the atomic structure of dislocation cores is further enhanced if the directional covalent component of the bonding is significant. The detailed behaviour of core structures of dislocations can be extremely complex and the most direct way of studying such structures, related models of dislocation motion, formation and propagation of twins, as well as the interaction of dislocations and twins with lamellar interfaces is by means of atomistic simulations. Likewise, atomic level studies are indispensable in studies of diffusion mechanisms in Ti-Al-based compounds, both in the bulk and at interfaces. These investigations are crucial for fundamental understanding of formation and hightemperature stability of the lamellar structure and its creep resistance (Mishin and Herzig 2000).

An appropriate description of atomic interactions is an essential prerequisite for a physically meaningful atomistic level modelling. In recent years, several centralforce potentials of the Finnis-Sinclair (FS) (1984) (Girshick and Vitek 1995, Vitek et al. 1997a, Paidar et al. 1999) and embedded-atom method (EAM) type (Daw and Baskes 1984, Rao et al. 1991, Farkas 1994, Simmons et al. 1993, 1997) were constructed and employed in studies of dislocations, interfaces and point defects. These calculations revealed a number of general features of lattice defects that can be 
expected in the $\mathrm{L} 1_{0}$ structure but obviously neglect the strong covalent and directional bonding that is characteristic for the titanium aluminides, as documented by a number of density functional theory (DFT)-based calculations (Fu and Yoo 1990, 1992, Woodward et al. 1992, Song et al. 1994, Nguyen-Manh et al. 1995, Zou et al. 1995, Nguyen-Manh and Pettifor 1999a,b). This pitfall of central forces is reflected, for example, in too low energies of stacking faults, in particular the superlattice intrinsic stacking fault which is analogous to the intrinsic stacking fault in fcc materials (Paidar et al. 1999). The reason is that, in any central-force scheme, only thirdneighbour and other more distant-neighbour interactions contribute to this energy. A higher energy of this fault can be attained if the central force potentials are longer ranged (Rao et al. 1991, Farkas 1994, Simmons et al. 1993, 1997, Panova and Farkas 1998). However, the DFT calculations (Woodward et al. 1992, Woodward and Maclaren 1996, Vitek et al. 1997b, Ehmann and Fähnle 1998) show that the true physical reason for the high value of the stacking-fault energy is the covalent bonding. It is also well known that the central-force schemes cannot predict negative Cauchy pressures $C_{13}-C_{44}=-0.213 \mathrm{eV}^{-3}$ and $C_{12}-C_{66}=-0.04 \mathrm{eV}^{-3}$ (Tanaka et al. 1996a) in TiAl, if physically motivated embedding functions are used.

While the DFT is at present the state of the art, and corresponding calculations lead to correct predictions for elastic moduli, stacking-fault energies and other physical quantities, it is severely limited by the number of independent atoms that can be treated in such studies. Simulations of dislocations, interfaces and their interactions require a minimum of several thousands of atoms in the relaxed block while 100200 atoms is at present the maximum in most DFT calculations. For this reason, various approximate schemes that include non-central forces have been advanced in recent years. Examples are approaches based on the tight-binding (TB) method within the approximation of low-order moments of the electronic density of states (Carlsson 1991, Foiles 1993) and on the perturbation expansions employed in the framework of first-principles generalized pseudopotential theory (Moriarty 1990, Moriarty and Widom 1997, Widom et al. 2000).

In this paper, we use the interatomic bond-order potentials (BOPs) in which the required covalent bonding is included explicitly within the TB approximation. This method, using an orthogonal basis and two-centre bond integrals, was formulated earlier by one of us (Pettifor 1989) and further developed (Aoki 1993, Aoki and Pettifor 1993, 1994) and implemented in the Oxford order- $N$ package (OXON) (Horsfield et al. 1996a,b). The primary advantage of BOPs is that calculations are performed in real space so that the large number of atoms that are needed to study extended defects can be simulated. The forces on the atoms are evaluated using the Hellmann-Feynman theorem.

Unfortunately and suprisingly, BOPs which were based on the conventional TB scheme were also not able to reproduce the negative Cauchy pressures for many materials, namely $C_{12}-C_{44}<0$ for cubic materials, or $C_{13}-C_{44}<0$ and $C_{12}-C_{66}<0$ for tetragonal or hexagonal materials (Nguyen-Manh et al. 1998). This problem of negative Cauchy pressures is ubiquitous because most high-temperature intermetallics composed of transition metals with partly or nearly half-filled $\mathrm{d}$ bands and typical sp-valent elements have negative Cauchy pressures (Tanaka and Koiwa 1996). For the fcc-like materials, such as TiAl, this particular property of elastic constants can be in turn related to the high ratio of $\mu / B$, where $\mu$ is the shear modulus on the slip plane and $B$ is the elastic bulk modulus (Pugh 1954, Cottrell 1998). From the modelling point of view, a negative Cauchy pressure reflects some 
important features in the nature of the bonding at the atomistic level. As has been explained elsewhere (Nguyen-Manh et al. 1998), the failure of BOPs to yield negative Cauchy pressures resides in the fact that the repulsive interaction within the conventional TB approximation is not screened by the local environment but is assumed to be simple pairwise. The purpose of this paper is, therefore, to construct BOPs for titanium aluminides in which the environmental dependence will be included in the repulsion for producing correctly negative Cauchy pressures.

The background for the construction of environmentally dependent BOPs for the $\mathrm{Ti}-\mathrm{Al}$ system will be presented in $\S 2$. We use a simple pd TB model in which only the $\mathrm{d}$ valence electrons on $\mathrm{Ti}$ and the $\mathrm{p}$ valence electrons on $\mathrm{Al}$ are retained explicitly in the attractive bond contribution. The input data of bonding integrals of $d-d, p-p$ and $\mathrm{d}-\mathrm{p}$ types and their functional form have been deduced using the first-principles TB linear muffin-tin orbitals (LMTO) method (Andersen and Jepsen 1984, Andersen et al. 1985). The very important property of these screened bond integrals, their transferability within the Ti-Al system, is thoroughly investigated in this paper. As mentioned earlier, a significant development in this study is inclusion of a many-body central force repulsive interaction that approximates the environmentally dependent overlap repulsion which is experienced by the valence $\mathrm{s}$ and $\mathrm{p}$ electrons. This addition to the energy is crucial for reproducing negative Cauchy pressures. Determination of the various parameters entering BOPs for TiAl via fitting to known equilibrium properties and certain results of DFT-based calculation are presented in $\S 3$. Testing of the constructed BOPs by comparing the calculations employing these potentials with available DFT-based calculations is summarized in $\S 4$. This involves comparison of the $\mathrm{L} 1_{0}$ TiAl with alternative structures possessing the same stoichiometry, investigation of large homogeneous distortions of the $\mathrm{L}_{0}$ structure, evaluation of the energies of stacking-fault-like defects, $\gamma$ surfaces describing large shearing along crystallographic planes, interfaces encountered in the lamellar TiAl and point defects (vacancies and antisites) in this compound. We also show that the constructed BOPs are transferable to different compositions, and in particular $\mathrm{Ti}_{3} \mathrm{Al}$ for which they predict, in agreement with experiment, that the lowestenergy structure is the hexagonal $\mathrm{D} 0_{19}$. Atomistic studies of dislocations in TiAl employing the constructed BOPs will be presented in a forthcoming paper. Finally, $\S 5$ contains several conclusions regarding the BOPs for TiAl.

\section{§2. Contributions to THE Bond-ORder POTEnTials}

\subsection{The pd tight-binding model}

The experimental structural trends within transition-metal-metalloid binary compounds are well described by a simple TB model in which only the d valence electrons on the transition-metal atoms and the $\mathrm{p}$ valence electron on the metalloid atoms are retained explicitly in the attractive TB contribution (Pettifor and Podloucky 1984, Pettifor 1995). A systematic study of the phase stability predictions for AB transition-metal aluminides using DFT within the local-density approximation (Nguyen-Manh et al. 1995, Nguyen-Manh and Pettifor 1999b) has also confirmed the validity of the pd TB model. We have, therefore, approximated the binding energy of the titanium aluminides in the following form:

$$
U=U_{\text {bond }}+U_{\text {env }}+U_{\text {pair }}
$$


where $U_{\text {bond }}$ is the attractive bond contribution arising within this pd TB model, $U_{\text {env }}$ is a repulsive environmentally dependent contribution arising from the strong core repulsion which the valence sp electrons experience in transition metals and their alloys (Pettifor 1978) and $U_{\text {pair }}$ is the usual pairwise contribution arising from the overlap repulsion and electrostatic interaction between the atoms (Sutton et al. 1988). We shall now discuss each of these three terms in turn.

\subsection{The bond energy}

Within the two-centre, orthogonal TB model the bond energy can be considered as a sum of contributions $U_{\text {bond }}^{i j}$ from individual $i-j$ bonds (for example Pettifor (1995)) so that

$$
U_{\text {bond }}=\frac{1}{2} \sum_{i, j \neq i} U_{\text {bond }}^{i j},
$$

where the individual contribution from the $i j$ th bond is given by

$$
U_{\text {bond }}^{i j}=\sum_{\alpha \beta} 2 H_{i \alpha, j \beta} \Theta_{j \beta, i \alpha},
$$

where the prefactor 2 accounts for spin degeneracy, and $H_{i \alpha, j \beta}$ and $\Theta_{j \beta, i \alpha}$ are the Hamiltonian and bond-order matrix elements that are associated with an individual bond $i-j$ and the corresponding atomic orbitals $\alpha$ and $\beta$. The bond integrals that enter the TB Hamiltonian are obtained directly from calculations employing screened TB LMTO theory (Andersen and Jepsen 1984, Andersen et al. 1985, Nakamura 1999, Nakamura et al. 2000). The angular dependence is of SlaterKoster form and their radial dependence is shown in figure 1 (see also Znam et al. 2000) for the dd, pp and dp bond integrals as a function of the bond length not only for $\mathrm{L1}_{0} \mathrm{TiAl}$ but also for $\mathrm{D}_{19} \mathrm{Ti}_{3} \mathrm{Al}$ and elemental hep Ti and fcc Al. It is seen that the bond integrals exhibit excellent transferability between different structures and compositions: $d d \sigma$ and $d d \pi$ for Ti-Ti bonding ( $d d \delta$ is not included in figure 1 but displays similar behaviour), $p p \sigma$ and $p p \pi$ for $\mathrm{Al}-\mathrm{Al}$ bonding, and $d p \sigma$ and $d p \pi$ for $\mathrm{Ti}-$ $\mathrm{Al}$ bonding. A salient feature of the $\mathrm{Ti}-\mathrm{Al}$ alloys, as well as of the pure titanium and aluminium, is that their structures are close packed, so that their second-nearestneighbour spacing is much larger than that of the first-nearest neighbours.

The bond integrals can be represented by a power-law dependence, namely

$$
\begin{aligned}
& d d \tau(R)=d d \tau_{0}\left(\frac{R_{0}^{\mathrm{Ti}-\mathrm{Ti}}}{R}\right)^{n_{\mathrm{Ti}-\mathrm{Ti}}} \\
& p p \tau(R)=p p \tau_{0}\left(\frac{R_{0}^{\mathrm{Al}-\mathrm{Al}}}{R}\right)^{n_{\mathrm{Al}-\mathrm{Al}}}, \\
& d p \tau(R)=d p \tau_{0}\left(\frac{R_{0}^{\mathrm{Ti}-\mathrm{Al}}}{R}\right)^{n_{\mathrm{T}-\mathrm{Al}}}
\end{aligned}
$$

where $\tau=\sigma$ and $\pi$ or $\delta$ and $R_{0}^{\mathrm{Ti}-\mathrm{Ti}}$ denotes the nearest $\mathrm{Ti}-\mathrm{Ti}$ spacing in hep Ti $\left(2.950 \AA\right.$ ) , while $R_{0}^{\mathrm{Al}-\mathrm{Al}}$ and $R_{0}^{\mathrm{Ti}-\mathrm{Al}}$ denote the nearest $\mathrm{Al}-\mathrm{Al}$ and $\mathrm{Ti}-\mathrm{Al}$ spacings in TiAl $(2.832 \AA$ and $2.8547 \AA$ respectively). It follows from TB LMTO calculations that the ratios of the bond integrals are not canonical but take the screened values which are as follows: $d d \sigma=-1.1526 \mathrm{eV}, d d \pi=0.5284 \mathrm{eV}, d d \delta=-0.0622 \mathrm{eV}$, $p p \sigma=1.8986 \mathrm{eV}, p p \pi=-0.2255 \mathrm{eV}, d p \sigma=-1.3970 \mathrm{eV}$ and $d p \pi=0.3748 \mathrm{eV}$. Since 

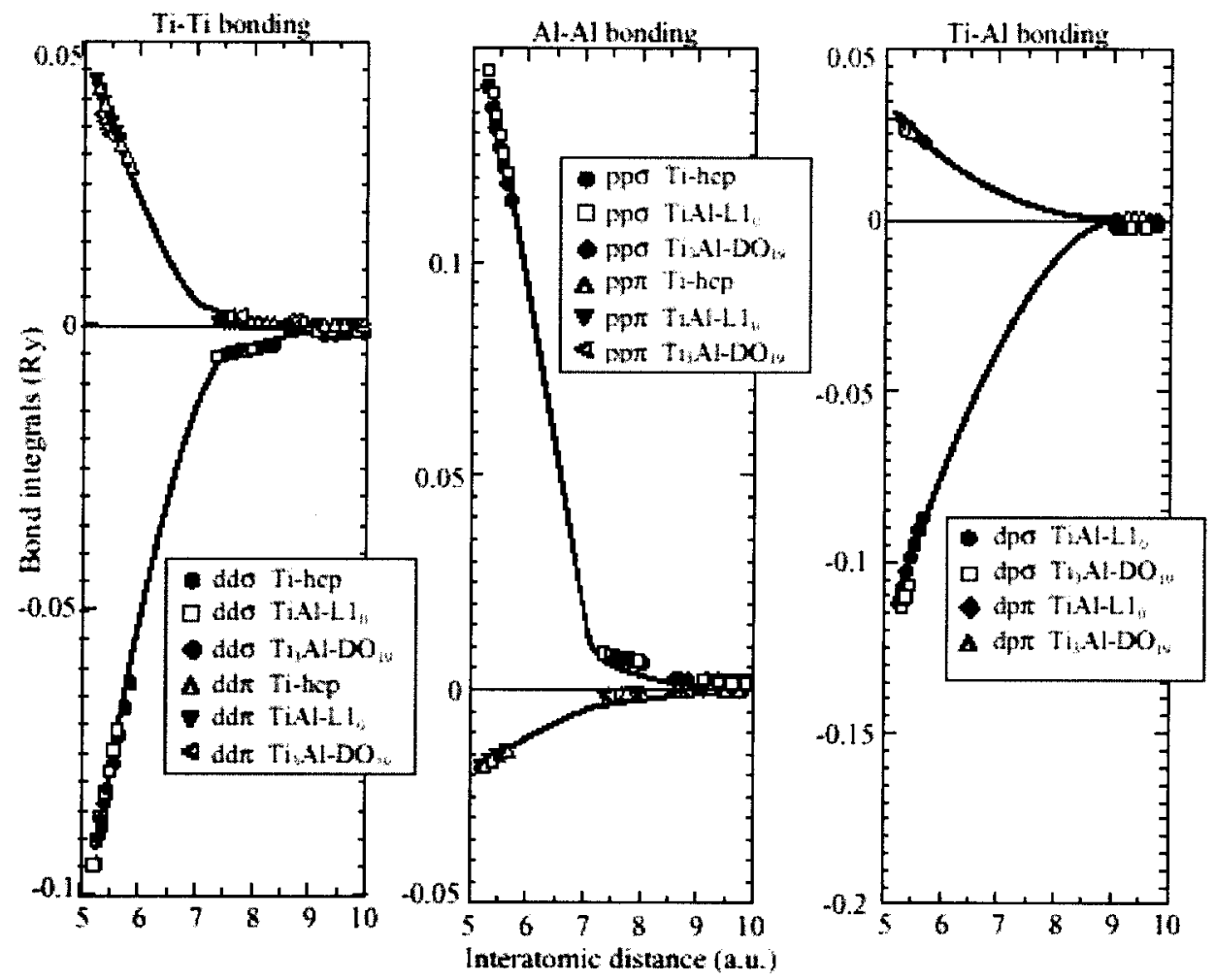

Figure 1. Bonding integrals in $\mathrm{Ti}-\mathrm{Al}$ alloys and pure $\mathrm{Ti}$ and $\mathrm{Al}$ as functions of interatomic distances calculated using the TB LMTO method (symbols) and analytical fittings described in the text (-).

the bond integrals fall off rapidly with increasing distance, we have cut them off at $R^{\text {cut }}=4.8 \AA$, that is just before the third-nearest neighbours in TiAl (about $4.92 \AA$ ). In order to avoid any discontinuity at the cut-off, the bond integrals are represented by a polynomial of fifth order for $R_{1}<R<4.8 \AA$ such that their magnitude and first and second derivatives are continuous at $R_{1}$ and zero for $R=R^{\mathrm{cut}}\left(R_{1}=3.1 \AA\right.$ for $\mathrm{Ti}-\mathrm{Ti}$ and $\mathrm{Al}-\mathrm{Al}$ interactions and 3.0 $\mathrm{A}$ for Ti-Al interactions). We should note that this pairwise-like behaviour of the screened matrix elements in close-packed titanium aluminides is in marked contrast with intermetallics such as $\mathrm{MoSi}_{2}$ (Nguyen-Manh et al. 2000). The latter is based on an underlying bcc lattice in which the secondnearest neighbours are only $14 \%$ further away than the first. This leads to a strong discontinuity between the bond integrals for the first- and second-nearest-neighbour shells, which requires the introduction of an explicit environmentally dependent screening (Hass et al. 1998, Nguyen-Manh et al. 2000).

The bond-order matrix elements, which determine the bond energies through equation (3), are obtained from BOP theory using the OXON package (Horsfield et al. 1996a,b). This real-space method requires the introduction of a fictitious temperature in order to damp down the long-range Friedel oscillations in metals, thereby guaranteeing the validity of the Hellmann-Feynman theorem when evaluating forces (Horsfield and Bratkovsky 1996, Girshick et al. 1998). We have found that sufficient accuracy is provided by $k_{\mathrm{B}} \mathrm{T}=0.3 \mathrm{eV}$ if $\mathrm{BOP}$ theory retains contributions 
up to the ninth moment in the local electronic density of states, or equivalently five levels in the continued-fraction representation for the corresponding Green's function. The individual forces on all the atoms in our calculations are converged to better than $0.01 \mathrm{eV}^{-1}$. The bond-order matrix is evaluated under the assumption of local charge neutrality ( $\mathrm{LCN}$ ) which is an excellent approximation for metallic systems. This is achieved by adjusting self-consistently the on-site atomic energy levels $\epsilon_{\mathrm{d}}$ on Ti sites and $\epsilon_{\mathrm{p}}$ on $\mathrm{Al}$ sites; this procedure is an integral part of the OXON package. We find in TB LMTO calculations that in the perfectly stoichiometric alloys $\mathrm{TiAl}$ and $\mathrm{Ti}_{3} \mathrm{Al}$ the self-consistent values of the atomic energy level difference $\epsilon_{\mathrm{p}}-\epsilon_{\mathrm{d}}$ are $2.244 \mathrm{eV}$ and $1.915 \mathrm{eV}$ respectively. These values have been used as starting on-site energy differences within the BOP scheme but owing to the LCN condition they are not the same in final self-consistent calculations. In particular, for non-equivalent atoms of the same type, which are always present when studying defective structures, the self-consistent values of on-site atomic energy levels are generally different. Importantly, because of LCN, there are no ionic or Madelung contributions entering the binding energy (1).

\subsection{The environmentally dependent core repulsion}

Although in the previous section the transition-metal atoms were characterized by only their valence $d$ electron contribution, their valence sp electrons play a critical role in providing the necessary repulsion to counter the attractive bond term. This arises from the strong repulsion as they are squeezed into the ion core regions under the influence of the large covalent bonding forces (Pettifor 1978). We, therefore, represent this repulsion by a screened Yukawa-type potential (Nguyen-Manh et al. 1998):

$$
U_{\mathrm{env}}=\frac{1}{2} \sum_{i, j \neq i} \frac{B}{R_{i j}} \exp \left[-\lambda_{i j}\left(R_{i j}-2 R_{\mathrm{c}}\right)\right]
$$

where $R_{\mathrm{c}}$ is the core radius and $\lambda_{i j}=\frac{1}{2}\left(\lambda_{i}+\lambda_{j}\right)$. The screening exponent $\lambda_{i j}$ is dependent on the local density and environment of atoms $i$ and $j$. This can be shown either within the nearly free-electron approximation (for example Pettifor and Ward (1984)) or within a variationally determined TB approximation (Skinner and Pettifor 1991). Here we model the environmental dependence through an embedded-atom-type expression by writing

$$
\lambda_{i}=\lambda_{0}+\left(\sum_{k \neq i} C \exp \left(-\nu R_{i k}\right)\right)^{1 / m},
$$

where $\lambda_{0}$ (the unscreened value of the exponent), $C, \nu$ and $m$ are the adjustable parameters. These parameters are different for different chemical species and determined by fitting the experimental values of the Cauchy pressures $C_{13}-C_{44}$ and $C_{12}-C_{66}$ in hep Ti and $\mathrm{L}_{0}$ TiAl. Since in equilibrium the pairwise term in equation (1) does not contribute implicitly to these pressures (Sob and Vitek 1996, NguyenManh et al. 1998) and the bond contribution is fixed and computed directly from the variation of bond integrals and bond orders with strain, these two Cauchy pressures can be written as

$$
C_{13}-C_{44}=\left(C_{13}-C_{44}\right)^{\text {bond }}+\left(C_{13}-C_{44}\right)^{\text {env }}+\frac{1}{4}\left(\sigma_{11}^{\text {bond }}+\sigma_{33}^{\text {bond }}+\sigma_{11}^{\text {env }}+\sigma_{33}^{\text {env }}\right)
$$

and 


$$
C_{12}-C_{66}=\left(C_{12}-C_{66}\right)^{\text {bond }}+\left(C_{12}-C_{66}\right)^{\text {env }}+\frac{1}{2}\left(\sigma_{11}^{\text {bond }}+\sigma_{11}^{\text {env }}\right) .
$$

$\sigma_{\mu \nu}$ are the appropriate stress components defined as

$$
\sigma_{\mu \nu}=\frac{1}{\Omega} \sum_{i, k>i} \frac{\partial U}{\partial R_{i k}^{\mu}} R_{i k}^{\nu},
$$

where $\Omega$ is the volume of the system. We shall see in the next section that not only are the bond contributions $\left(C_{13}-C_{44}\right)$ bond and $\left(C_{12}-C_{66}\right)$ bond positive but also so are the bond contributions coming from the stress $\sigma_{11}^{\text {bond }}$ and $\sigma_{33}^{\text {bond }}$ (Nguyen-Manh et al. 1998). However, we find that the environmental dependence in the screening exponent gives rise to negative contributions of $\left(C_{13}-C_{44}\right)^{\text {env }},\left(C_{12}-C_{66}\right)^{\text {env }}, \sigma_{11}^{\text {env }}$ and $\sigma_{33}^{\text {env }}$, thereby allowing the observed negative Cauchy pressures to be fitted.

\subsection{The pairwise potential}

The final contribution to the binding energy $U_{\text {pair }}$ arises nominally from the overlap repulsion and the electrostatic interaction between the atoms and it can be expressed as

$$
U_{\text {pair }}=\frac{1}{2} \sum_{i \neq j} V\left(R_{i j}\right)
$$

where $V\left(R_{i j}\right)$ is a pair potential. For this pair-potential we employ here the same functional form as that used by Ackland et al. (1987) when constructing FinnisSinclair potentials, namely a sum of cubic splines:

$$
V\left(R_{i j}\right)=\sum_{k} A_{k}\left(R_{k}-R_{i j}\right)^{3} H\left(R_{k}-R_{i j}\right),
$$

with $H(x)$ the Heaviside step function. The node points of the splines $R_{k}$ and the coefficients $A_{k}$ are different for Ti-Ti, $\mathrm{Al}-\mathrm{Al}$ and Ti-Al pair interactions. This functional form assures that $V\left(R_{i j}\right)$ and its first and second derivatives are everywhere continuous and equal to zero at the cut-off. The parameters in this potential are obtained by fitting to those elastic constants that remain after fixing the Cauchy pressure and to the cohesive energy and equilibrium lattice parameters.

\section{§3. Constructions of ENVIRONMENTALly DEPENDENT BOND-ORDER POTENTIALS FOR TiAl}

\subsection{Fitting procedure}

The energy given by equation (1) can be separated into three parts that correspond to $\mathrm{Ti}-\mathrm{Ti}, \mathrm{Al}-\mathrm{Al}$ and $\mathrm{Ti}-\mathrm{Al}$ interactions. We start with the construction of the Ti-Ti BOP by fitting the equilibrium of pure Ti. Ideally, this part of the binding energy should be usable directly in $\mathrm{Ti}-\mathrm{Al}$ alloys since, as shown in the previous section, the bond integrals $d d \tau$ (equation (4)) are fully transferable between $\mathrm{Ti}$, $\mathrm{TiAl}$ and $\mathrm{Ti}_{3} \mathrm{Al}$. In practice, however, as we shall see later, the BOP constructed for Ti can only be employed as a part of the BOPs for TiAl when additional, albeit small, adjustments are made.

Recently a BOP for pure Ti was developed by Girshick et al. 1998. However, this BOP did not contain the environmentally dependent repulsion and suffered, therefore, the shortcoming that the Cauchy pressures were not accurately reproduced (though they are both positive in hep Ti) showing errors of $50 \%$ and $100 \%$ in 
$C_{12}-C_{66}$ and $C_{13}-C_{44}$ respectively. Here we have included the environmentally dependent repulsion $E_{\text {env }}$ and have constructed a new BOP for the Ti-Ti interaction by employing the following sequential fitting procedure. The first step, the construction of the bond part, employs the power law for the bond integrals (equation (4)) with $R_{0}^{\mathrm{Ti}-\mathrm{Ti}}$ taken as the nearest-neighbour spacing in the basal plane of hcp $\mathrm{Ti}$ $(2.950 \AA)$ and the scaling exponent $n_{\mathrm{Ti}-\mathrm{Ti}}$ representing the distance dependence of the radial part of the bond integrals between Ti and Ti. As mentioned in the previous section, these integrals are cut off smoothly at $R^{\text {cut }}=4.8 \AA$. Five recursion levels of continued fractions were used when calculating $U_{\mathrm{bond}}^{\mathrm{Ti}-\mathrm{Ti}}$. The number $N_{\mathrm{d}}$ of electrons in the $\mathrm{d}$ band (i.e. d-band filling) and the scaling exponent $n_{\mathrm{Ti}-\mathrm{Ti}}$ were adjusted so as to assure the correct energy difference between the two close-packed structures, hcp and fcc ( $58 \mathrm{meV}^{-1}$ atom $^{-1}$ according to an ab-initio fully linear augmented plane-wave (FLAPW) value), while reproducing closely the TB LMTO values of the bond integrals shown in figure 1 . This led to $N_{\mathrm{d}}=2.2$ and $n_{\mathrm{Ti}-\mathrm{Ti}}=3.9$ with an energy difference between hcp and fcc structures of $52.2 \mathrm{meV}$ atom $^{-1}$. Note that both these parameters have to be further optimized for the use in TiAl, as explained below.

The second step of the fitting procedure is to optimize the parameters of the environmentally dependent repulsive term given by equations (5) and (6) so as to reproduce the Cauchy pressures in hcp Ti which are $C_{12}-C_{66}=0.261 \mathrm{eV}^{-3}$ and $\left.C_{13}-C_{44}\right)=0.109 \mathrm{eV}^{-3}$. In order to simplify the fitting procedure we use $m=2$ and $R_{\mathrm{c}}=1 \AA$ for the core radius of Ti atoms, but note that the value of $R_{\mathrm{c}}$ is optimized for the Ti-Ti BOP in TiAl. The dependence of the Cauchy pressure on the parameters $\mathrm{C}, \nu$ and $\lambda_{0}$ is complex and highly nonlinear, but numerical evaluation of the environmental repulsive contribution to the Cauchy pressures for a given set of parameters is straightforward and fast. This allowed us to determine simultaneously these three parameters within given ranges while adjusting the prefactor $B$. In this way, we were able to fit the Cauchy pressures to within about $10 \%$. The corresponding values of these parameters are summarized in table 1 . The expression for $\lambda_{i}$, given by equation (6), was cut off at $R_{i j}=5.4 \AA$, just beyond the fifth-nearest neighbours in hep Ti but only beyond the third-nearest neighbours in TiAl. For this purpose the term $\exp \left(-\nu R_{i k}\right)$ was replaced by a fifth-order polynomial in $R_{i k}$ for

Table 1. Parameters of the environmentally dependent repulsive term in BOPs for $\mathrm{TiAl}$, given by equations (5) and (6). Values of the parameters for the $\mathrm{Ti}-\mathrm{Al}$ interactions are calculated as $B^{\mathrm{Ti}-\mathrm{Al}}=\left(B^{\mathrm{Ti}-\mathrm{Ti}} B^{\mathrm{Al}-\mathrm{Al}}\right)^{1 / 2}$ and $R_{\mathrm{c}}^{\mathrm{Ti}-\mathrm{Al}}=\frac{1}{2}\left(R_{\mathrm{c}}^{\mathrm{Ti}-\mathrm{Ti}}+R_{\mathrm{c}}^{\mathrm{Al}-\mathrm{Al}}\right) ; \nu, \lambda_{0}$ and $m$ are then needed only for $\mathrm{Al}-\mathrm{Al}$ and $\mathrm{Ti}-\mathrm{Ti}$ interactions.

\begin{tabular}{lcc}
\hline & $\mathrm{Al}-\mathrm{Al}$ & $\mathrm{Ti}-\mathrm{Ti}$ \\
\hline$B(\mathrm{eV})$ & $10^{5}$ & 183.0 \\
$C\left(\AA^{-2}\right)$ & 210.0 & 175.0 \\
$\nu\left(\AA^{-1}\right)$ & 2.79 & 1.90 \\
$\lambda_{0}\left(\AA^{-1}\right)$ & 3.308 & 3.900 \\
$R_{\mathrm{c}}(\AA)$ & 0.25 & 0.90 \\
$m$ & 2.0 & 2.0 \\
\hline
\end{tabular}


$4.8 \AA<R_{i k}<5.4 \AA$ in order to avoid any discontinuity at the cut-off. Analogously, for any given value of $\lambda_{i j}$ the cut-off at $R_{i j}=5.4 \AA$ was introduced in equation (5) such that the exponential term was replaced by a fifth-order polynomial in $R_{i j}$ for $4.8 \AA<R_{i j}<5.4 \AA$.

Finally, the parameters of the pair potential term (equation (11)) were determined so as to reproduce the cohesive energy of $4.85 \mathrm{eV} \mathrm{atom}^{-1}$ of the hcp Ti, the equilibrium lattice parameters $a=2.95 \AA$ and $c=4.683 \AA$ and the three remaining elastic constants $C_{13}=0.426 \mathrm{eV}^{-3}, C_{33}=1.189 \mathrm{eV}^{-3}$ and $C_{44}=0.317 \mathrm{eV}^{-3}$. The values of the node points $R_{k}$ and coefficients $A_{k}$ are summarised in table 2 . Owing to its form as a sum of cubic splines the pair potential is cut off smoothly at $R^{\text {cut }}=4.8 \AA$. We should note that, in the old version of the Ti BOP (Girshick et al. 1998), the pair potential is supplemented by an additional repulsive term for atomic separations below the equilibrium nearest-neighbour spacing $(2.950 \AA)$ in order to avoid collapse of the lattice being driven by the bond part. In the present case, however, the environmental repulsive term provides sufficient repulsion to compensate the power law attraction from the bond energy.

The construction of the BOPs describing the $\mathrm{Ti}-\mathrm{Al}$ and $\mathrm{Al}-\mathrm{Al}$ interactions was carried out similarly as for the Ti-Ti interaction. The first step was again construction of the bond parts which now involve the bond integrals $d p \sigma(R), d p \pi(R)$ and $p p \sigma(R)$ and $p p \pi(R)$ respectively. They are represented by the power law according to equation (4) with $R_{0}^{\mathrm{Ti}-\mathrm{Al}}$ and $R_{0}^{\mathrm{Ti}-\mathrm{Al}}$ denoting the nearest Ti-Al and $\mathrm{Al}-\mathrm{Al}$ spacings in TiAl (2.8320 $\AA$ and $2.8547 \AA$, respectively). The values of the coefficients $d p \tau_{0}$ and $p p \tau_{0}$ were determined from the results of ab-initio TB LMTO calculations and are presented in $\S 2.2$. As already mentioned, all the bond integrals are cut off smoothly at $R^{\text {cut }}=4.8 \AA$. The power-law parameters $n_{\mathrm{Ti}-\mathrm{Al}}$ and $n_{\mathrm{Al}-\mathrm{Al}}$ were optimized to assure correct ordering of the two competing structures, the fcc-based $\mathrm{L}_{0}$ and the hexagonal B19 (Nguyen-Manh et al. 1995, Nguyen-Manh and Pettifor 1999b), while reproducing closely the ab-initio calculated values of the bond integrals shown in figure 1. The energy difference between these two structures, determined by fully relaxed ab-initio calculation using the CASTEP (1999) method, is $32 \mathrm{meV}$ atom $^{-1}$.

When calculating the energy of the B19 structure the atoms within the unit cell have to be relaxed. This cannot be achieved using the bond part alone but all contributions to the binding energy have to be included. After an exhaustive analysis of the influence of different potential parameters on the energy difference between $\mathrm{L}_{0}$ and $\mathrm{B} 19$ structures we set this difference at $35.9 \mathrm{meV}$ atom $^{-1}$, very close to the value found by ab-initio calculations. This was attained for $n_{\mathrm{Ti}-\mathrm{Al}}=2.7, n_{\mathrm{Al}-\mathrm{Al}}=3.4$ and $N_{\mathrm{p}}=2.9$ but after adjusting simultaneously the parameters of the Ti-Ti inter-

Table 2. Parameters of the $\mathrm{Ti}-\mathrm{Ti}, \mathrm{Al}-\mathrm{Al}$ and the Ti-Al pair potentials in the BOP scheme for TiAl with $c / a=1.016(a=4.005 \AA ; c=4.06908 \AA)$. Note that the potential for Ti-Ti is in the case of $\mathrm{Ti}-\mathrm{Al}$ alloys cut off smoothly at $R^{\text {cut }}=4.5 \AA$.

\begin{tabular}{rrrrrrc}
\hline$k$ & \multicolumn{1}{c}{$A_{k}^{\mathrm{Ti}-\mathrm{Ti}}$} & $R_{k}^{\mathrm{Ti}-\mathrm{Ti}}$ & \multicolumn{1}{c}{$A_{k}^{\mathrm{Al}-\mathrm{Al}}$} & \multicolumn{1}{c}{$R_{k}^{\mathrm{Al}-\mathrm{Al}}$} & \multicolumn{1}{c}{$A_{k}^{\mathrm{Ti}-\mathrm{Al}}$} & $R_{k}^{\mathrm{Ti}-\mathrm{Al}}$ \\
\hline 1 & -17.5448512679974 & 4.80 & -0.3004998221986 & 5.30 & -0.2073837398907 & 5.30 \\
2 & 30.6156648774779 & 4.75 & 0.4729360381743 & 5.10 & 0.2741033033228 & 5.20 \\
3 & -10.9928131235048 & 4.70 & 21.7035240133815 & 3.00 & 2.7167981511420 & 3.00 \\
4 & -19.4079886940734 & 4.25 & 47.4918934741912 & 2.90 & & \\
5 & 17.5688073952306 & 4.20 & & & & \\
6 & 1.06358595447043 & 3.30 & & & & \\
\hline
\end{tabular}


action such that $N_{\mathrm{d}}=2.0, n_{\mathrm{Ti}-\mathrm{Ti}}=3.6$ and $R_{\mathrm{c}}^{\mathrm{Ti}-\mathrm{Ti}}=0.9 \AA$. The latter change is congruent with the fact that the nearest-neighbour Ti-Ti separation in TiAl is $2.832 \AA$ while it is $2.95 \AA$ in hcp Ti. However, we should note that the above change does not affect the energy ordering between the ground-state hcp $\mathrm{Ti}$ and the fcc and bcc structures; the fcc-hcp energy difference is $62 \mathrm{meV}$ atom $^{-1}$ while the bcc-hcp energy difference is $162 \mathrm{meV}$ atom $^{-1}$, which compares well with the full potential FP LMTO values (Nguyen-Manh et al. 1996) of $92 \mathrm{meV} \mathrm{atom}^{-1}$ and $128 \mathrm{meV}^{-1} \mathrm{atom}^{-1}$ respectively. The dependences of the bond integrals on $R$, evaluated according to equation (4) when using the above-mentioned parameters, are shown as solid curves in figure 1 .

The parameters of the repulsive environmentally dependent term (equations (5) and (6)) were optimized such as to reproduce the two negative Cauchy pressures for the $\mathrm{L}_{0}$ TiAl. To simplify the fitting, the cross-term parameters, namely $B^{\mathrm{Ti}-\mathrm{Al}}$ and $R_{\mathrm{c}}^{\mathrm{Ti}-\mathrm{Al}}$, were set equal to the geometric and arithmetic averages respectively of the corresponding parameters for the elements $\mathrm{Ti}$ and $\mathrm{Al}$. Thus only parameters for the environmental dependence of the $\mathrm{Al}-\mathrm{Al}$ interaction needed to be determined. Using $m=2$ and $R_{\mathrm{c}}^{\mathrm{Al}-\mathrm{Al}}=0.25 \AA$, the resulting Cauchy pressures for TiAl, calculated using the present BOPs, are $C_{13}-C_{44}=-0.206 \mathrm{eV}^{-3}$ and $C_{12}-C_{66}=-0.045 \mathrm{eV}^{-3}$ with the parameters of the environmentally dependent repulsive term for $\mathrm{Al}-\mathrm{Al}$ summarized in table 1 . For both $\mathrm{Ti}-\mathrm{Al}$ and $\mathrm{Al}-\mathrm{Al}$, the expression for $\lambda_{i}$, given by equation (6), was cut off at $R_{i k}=5.4 \AA$, as for Ti-Ti; this cut-off is just beyond the third-nearest neighbours in TiAl. An analogous cut-off was introduced for the exponential term in equation (5). We should, however, note here that the obtained parameters should not be used to represent a real interatomic potential for elemental $\mathrm{Al}$ as we did not fit them with any physical property of pure Al in the fcc ground state. Also a realistic interatomic potential for $\mathrm{Al}$ will require the bond energy contribution coming from both $\mathrm{s}$ and $\mathrm{p}$ valence electrons rather than only the $\mathrm{p}$ contribution.

Finally, the pair potentials describing the $\mathrm{Ti}-\mathrm{Al}$ and $\mathrm{Al}-\mathrm{Al}$ interactions were constructed so as to fit for TiAl the cohesive energy, the lattice parameters $a$ and $c$, and the four remaining elastic constants, $C_{11}, C_{33}, C_{44}$ and $C_{66}$; the two other elastic constants are determined by the Cauchy pressures that had already been fitted. For the Ti-Ti pair potential we use the same potential as for pure Ti (table 2) but cut off at $R^{\text {cut }}=4.5 \AA$ rather than $4.8 \AA$; this was again achieved by adding a polynomial which assures that no discontinuity occurs at $R^{\text {cut }}$. This shorter cut-off was essential for attaining the correct energy difference between $\mathrm{L1}_{0}$ and $\mathrm{B} 19$ structures. Since the Ti-Ti pair potential was fixed, the Ti-Al and Al-Al pair potentials were constructed so as to fit the seven quantities listed above. This was carried out by using in equation (11) four splines for $\mathrm{Al}-\mathrm{Al}$ and three splines for Ti-Al potentials. The corresponding spline coefficients $A_{k}^{\mathrm{Ti}-\mathrm{Al}}$ and $A_{k}^{\mathrm{Al}-\mathrm{Al}}$, as well as spline nodes $R_{k}^{\mathrm{Ti}-\mathrm{Al}}$ and $R_{k}^{\mathrm{Al}-\mathrm{Al}}$, are listed in table 2 . The pair potentials for the Ti-Ti, Ti-Al and $\mathrm{Al}-\mathrm{Al}$ interactions are shown in figure 2.

A comparison of experimental values of the cohesive energy, lattice parameters and elastic constants with those calculated using the constructed BOPs is presented in table 3 . None of the calculated values differs from experiment by more than $10 \%$, which is very adequate agreement for our purposes. Since an important aspect of the present scheme is the ability to reproduce the negative Cauchy pressures in $\mathrm{L} 1_{0} \mathrm{TiAl}$, different contributions to the two Cauchy pressures, defined by equations (7) and (8), are presented in table 4 . This demonstrates clearly that the environmentally depen- 


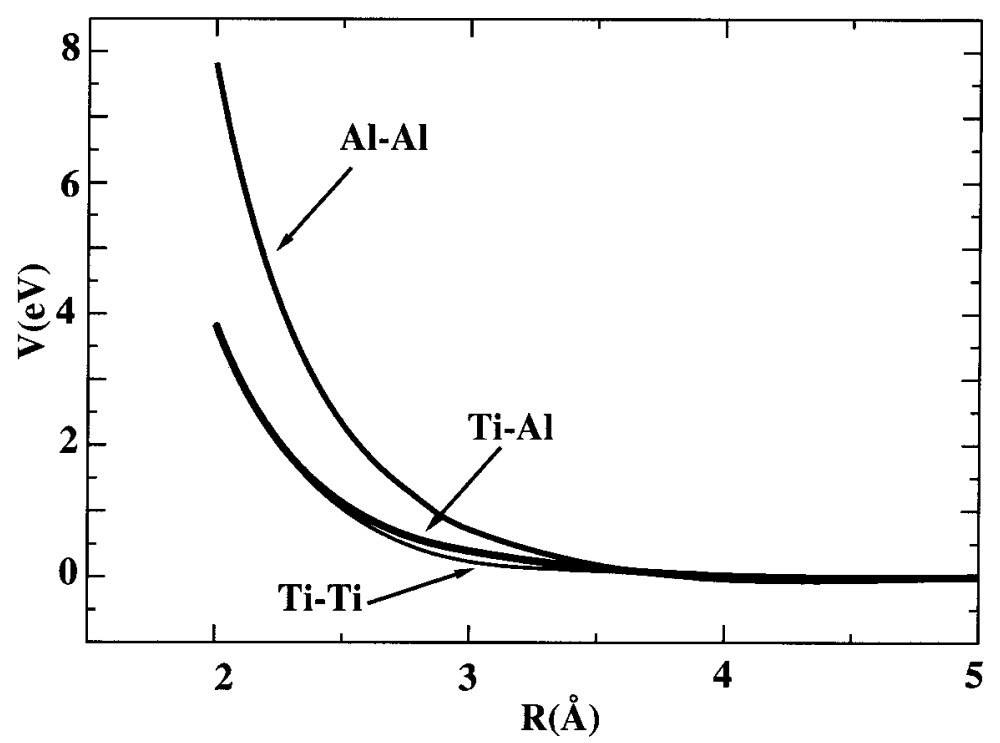

Figure 2. The $\mathrm{Ti}-\mathrm{Ti}, \mathrm{Ti}-\mathrm{Al}$ and $\mathrm{Al}-\mathrm{Al}$ pair potentials.

Table 3. Fitted equilibrium properties of $\mathrm{L}_{0}$ TiAl calculated using the present BOPs and compared with the corresponding experimental values. The cohesive energies have been taken from Hultgren et al. (1973), the lattice parameters $a$ and $c$ from Pearson (1967) and the elastic moduli from Tanaka et al. (1996a).

\begin{tabular}{lccc}
\hline & $\begin{array}{c}\text { Calculated from BOPs } \\
\text { for } c / a=1.016\end{array}$ & $\begin{array}{c}\text { Calculated from BOPs } \\
\text { for } c / a=1.0\end{array}$ & Experiment \\
\hline$E_{\text {coh }}(\mathrm{eV})$ & 4.520 & 4.520 & 4.520 \\
$a(\AA)$ & 4.005 & 4.025 & 4.005 \\
$c(\AA)$ & 4.069 & 4.005 & 4.069 \\
$C_{11}\left(\mathrm{eV}^{-3}\right)$ & 1.135 & 1.165 & 1.167 \\
$C_{33}\left(\mathrm{eV}^{-3}\right)$ & 1.136 & 1.132 & 1.136 \\
$C_{44}\left(\mathrm{eV}^{-3}\right)$ & 0.680 & 0.648 & 0.680 \\
$C_{12}\left(\mathrm{eV}^{-3}\right)$ & 0.427 & 0.433 & 0.467 \\
$C_{13}\left(\mathrm{eV}^{-3}\right)$ & 0.474 & 0.468 & 0.467 \\
$C_{66}\left(\mathrm{eV}^{-3}\right)$ & 0.473 & 0.507 & 0.507 \\
\hline
\end{tabular}

dent term gives the negative contributions resulting in the correct sign of the Cauchy pressures.

\subsection{The bond-order potential for TiAl with $c / a=1.0$}

When $c / a \neq 1$ there is a mismatch across the lamellar interfaces other than ordered twins (see $\S 4.3$ ) and this is compensated by misfit dislocations (Kad and Hazzledine 1992), the separation of which is about 50 lattice spacings for $c / a=1.016$ in $\mathrm{L}_{0}$ TiAl. While the period of such interfaces is the same as the separation of misfit dislocations, the region in between them is coherent and its structure is almost the same as if $c / a=1$. Hence, the coherent regions of lamellar 
Table 4. Contributions (from equations (7) and (8)) to the Cauchy pressures for TiAl within two BOP models. Experimental values are taken from Tanaka et al. (1996a).

\begin{tabular}{lccc}
\hline $\begin{array}{l}\text { Cauchy pressure } \\
\left(\mathrm{eV}^{-3}\right)\end{array}$ & $\begin{array}{c}\text { BOP } \\
(c / a=1.016)\end{array}$ & $\begin{array}{c}\text { BOP } \\
(c / a=1.000)\end{array}$ & Experiment \\
\hline$C_{13}^{\text {bond }}-C_{44}^{\text {bond }}$ & 0.056 & 0.093 & - \\
$C_{13}^{\text {env }}-C_{44}^{\text {env }}$ & -0.479 & -0.487 & - \\
$\frac{1}{4}\left(\sigma_{11}^{\text {bond }}+\sigma_{33}^{\text {bond }}\right)$ & 0.251 & 0.249 & - \\
$\frac{1}{4}\left(\sigma_{11}^{\text {env }}+\sigma_{33}^{\text {env }}\right)$ & -0.034 & -0.035 & - \\
$C_{13}-C_{44}$ & -0.206 & -0.180 & -0.213 \\
$C_{12}^{\text {bond }}-C_{66}^{\text {bond }}$ & 0.190 & 0.161 & - \\
$C_{12}^{\text {env }}-C_{66}^{\text {env }}$ & -0.504 & -0.495 & - \\
$\frac{1}{2} \sigma_{11}^{\text {bond }}$ & 0.306 & 0.297 & - \\
$\frac{1}{2} \sigma_{11}^{\text {env }}$ & -0.038 & -0.037 & -0.040 \\
$C_{12}-C_{66}$ & -0.046 & -0.074 & \\
\hline
\end{tabular}

Table 5. Parameters of the $\mathrm{Al}-\mathrm{Al}$ and the $\mathrm{Ti}-\mathrm{Al}$ pair potentials in the BOP for TiAl with ideal $c / a=1.0(a=c=4.0246 \AA)$.

\begin{tabular}{ccccc}
\hline$k$ & \multicolumn{1}{c}{$\begin{array}{c}A_{k}^{\mathrm{Al}-\mathrm{Al}} \\
\left(\mathrm{eV}^{-3}\right)\end{array}$} & $\begin{array}{c}R_{k}^{\mathrm{Al}-\mathrm{Al}} \\
\left(\mathrm{eV}^{-3}\right)\end{array}$ & $\begin{array}{c}A_{k}^{\mathrm{Ti}-\mathrm{Al}} \\
\left(\mathrm{eV}^{-3}\right)\end{array}$ & $\begin{array}{c}R_{k}^{\mathrm{Ti}-\mathrm{Al}} \\
\left(\mathrm{eV}^{-3}\right)\end{array}$ \\
\hline 1 & -0.19801917905365 & 5.30 & -0.20600926735481 & 5.30 \\
2 & 0.34709429667575 & 5.10 & 0.26921072547404 & 5.20 \\
3 & 25.41432898759122 & 3.00 & 2.39759314049279 & 3.00 \\
4 & -61.73913669578597 & 2.90 & & \\
\hline
\end{tabular}

interfaces, the period of which is the same as that of the crystal planes parallel to the interface, can be studied assuming that $c / a=1$. For this purpose, we have constructed another set of environmentally dependent BOPs for TiAl by modifying pair potentials describing the $\mathrm{Ti}-\mathrm{Al}$ and $\mathrm{Al}-\mathrm{Al}$ interactions so that the slightly different lattice parameters $a=c=4.0246 \AA$ are reproduced. This set of potentials uses the same bond-part and environmentally dependent term as for the case when $c / a=1.016$ as well as the same Ti-Ti pair potential. The new fitting parameters for the $\mathrm{Ti}-\mathrm{Al}$ and $\mathrm{Al}-\mathrm{Al}$ pair potentials are listed in table 5 and the cohesive energy, lattice parameters and elastic constants calculated using the BOPs for $c / a=1.0$ are presented in table 3. Corresponding contributions to the Cauchy pressures, defined by equations (7) and (8), which are now different owing to different values of $a$ and $c$, are presented in table 4 . It is again seen that the environmentally dependent term gives the negative contributions resulting in the negative sign of the predicted Cauchy pressures.

\section{$\S 4$. Testing OF THE TiAl BOND-ORDER POTENTIAL}

\subsection{Bulk properties}

The BOP for TiAl was constructed by fitting certain properties of the equilibrium $\mathrm{L} 1_{0}$ structure. Hence, it is important to test the applicability of this potential to 
atomic configurations with environments very different from that encountered in the ground-state $\mathrm{L} 1_{0}$ lattice. Our first calculation tests the stability of the $\mathrm{L}_{0}$ structure with respect to large homogeneous strain. This has been adhered by computing the binding energy as a function of homogeneous expansions and contractions of the two lattice parameters $a$ and $c$ by $\pm 20 \%$. The resulting energy surface, which is shown in terms of the contour map in figure 3 , possesses only one minimum, at the fitted equilibrium values of $a$ and $c$. Hence, within the tested deformation range, the potential does not lead to any instabilities or unphysical metastable configurations for non-uniform expansions or contractions of the lattice.

Our second calculation tests the relative order of different structures at the same composition as TiAl. The BOP predicts the ordering from $\mathrm{L}_{0} \rightarrow \mathrm{B} 19 \rightarrow \mathrm{B} 2 \rightarrow \mathrm{B} 1$ in agreement with ab-initio calculations (Nguyen-Manh et al. 1995, Nguyen-Manh and Pettifor 1999b). In addition, we have performed studies of tetragonal, trigonal and hexagonal transformation paths between the above structures (Nguyen-Manh et al. 1996, Sob et al. 1997, Paidar et al. 1999, Nguyen-Manh and Pettifor 1999a). Results of these calculations, which have been given by Znam et al. (2000) and Znam (2001), are in a satisfactory agreement with calculations performed using the ab-initio FLAPW method (Paidar et al. 1999).

Our final set of calculations tests the transferability of constructed BOPs for TiAl to the $3: 1$ stoichiometry of $\mathrm{Ti}_{3} \mathrm{Al}$, which crystallizes in the $\mathrm{D} 0_{19}$ structure. Table 6 shows the comparison between prediction and experimental (Tanaka et al. 1996b) and DFT-based calculated (Fu et al. 1995) cohesive energy and elastic constants. The

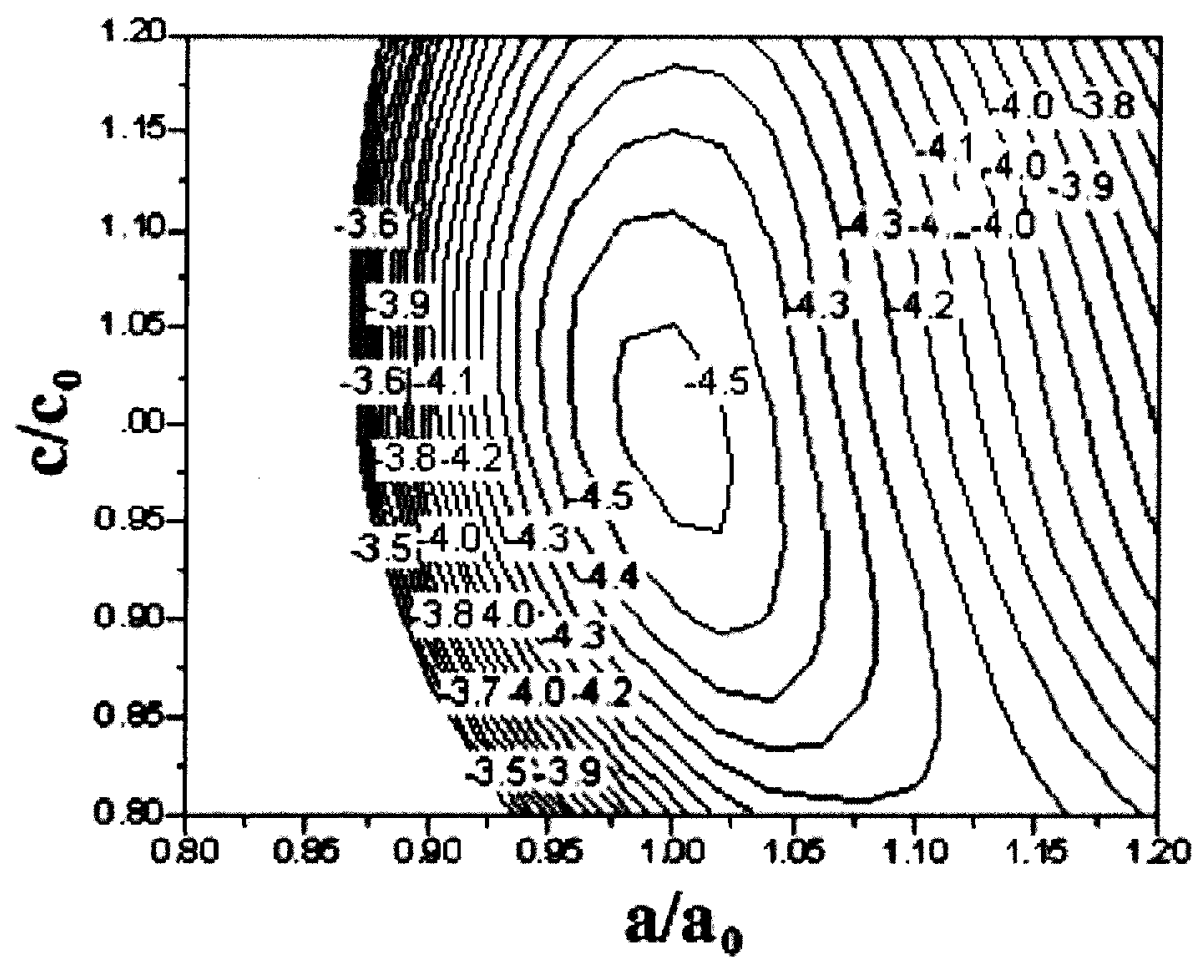

Figure 3. Energy surface (in electronvolts per atom) for large homogeneous deformation of the TiAl unit cell. 
Table 6. Equilibrium properties of $\mathrm{D}_{19}-\mathrm{Ti}_{3} \mathrm{Al}$, lattice parameters $a$ and $c$, cohesive energies and elastic constants, calculated using constructed BOPs and compared with experimental data (Tanaka et al. 1996b), and DFT-based calculations (Zou et al. 1995); the DFT cohesive energy value is taken from our FP LMTO calculations (see also van Schilfgaarde et al. (1991)).

\begin{tabular}{lccc}
\hline & BOP calculations & DFT & Experiment \\
\hline$a(\AA)$ & 5.568 & 5.640 & 5.780 \\
$c(\AA)$ & 4.634 & 4.568 & 4.647 \\
$c / a$ & 0.832 & 0.810 & 0.804 \\
Cohesive energy $\left(\mathrm{eV} \mathrm{atom}^{-1}\right)$ & 4.657 & 5.978 & 4.744 \\
$C_{11}\left(\mathrm{eV} \AA^{-2}\right)$ & 1.606 & 1.380 & 1.142 \\
$C_{12}\left(\mathrm{eV}^{-2}\right)$ & 0.896 & 0.433 & 0.556 \\
$C_{13}\left(\mathrm{eV}^{-2}\right)$ & 0.476 & 0.530 & 0.391 \\
$C_{33}\left(\mathrm{eV} \AA^{-2}\right)$ & 1.822 & 1.485 & 1.404 \\
$C_{44}\left(\mathrm{eV} \AA^{-2}\right)$ & 0.282 & 0.431 & 0.400 \\
$C_{66}\left(\mathrm{eV} \AA^{-2}\right)$ & 0.355 & 0.468 & 0.293 \\
\hline
\end{tabular}

agreement is very satisfactory considering that the titanium aluminide BOPs were developed by fitting to the alloy with the $1: 1$ stoichiometry. We have also calculated the energy difference between the two competing close packed structures: hexagonal $\mathrm{D} 0_{19}$ and cubic $\mathrm{Ll}_{2}$ and we find that $\mathrm{L}_{2}$ is predicted to be $4.2 \mathrm{meV}$ atom ${ }^{-1}$ higher than $\mathrm{D}_{19}$. This compares very well with the ab-initio calculated energy difference of $10 \mathrm{meV}$ atom $^{-1}$.

\section{2. $\gamma$ surface for $\{111\}$ planes and energies of stacking-fault-like defects}

Since close-packed $\{111\}$ planes are the slip and twinning planes in TiAl, the next important test of the BOPs is investigation of shearing along these planes and study of possible stacking-fault-like defects that participate in dissociation of dislocations on these planes. The stacking of three adjacent (111) planes in the $\mathrm{L} 1_{0}$ structure is shown in figure 4 . In this figure the vectors indicate three stacking-fault-like defects, formed by shifting the top layer (largest circles) with respect to the lower layers (small and intermediate circles) that are commonly assumed to exist on these planes. These are the antiphase domain boundary (APB) with the displacement $\frac{1}{2}[101]$, complex stacking fault (CSF) with the displacement $\frac{1}{2}[\overline{2} 11]$ and the superlattice intrinsic stacking fault (SISF) with the displacement $\frac{1}{6}[11 \overline{2}]$. The stability of these faults, as well the existence of other faults, can be investigated most comprehensively using the concept of the $\gamma$ surface, first introduced when searching for possible stacking faults in bcc metals (Vitek 1968). This surface is the plot of the energy of faults produced by various relative displacements of the two parts of a crystal cut along a chosen crystallographic plane. The local minima on this surface determine the displacement vectors of possible metastable stacking-fault-like defects, and the values of the energy at these minima are the energies of these faults. The $\gamma$ surface can be calculated relatively easily since the periodicity of the plane of the cut can be utilized and only relaxation perpendicular to the fault has to be carried out; no relaxation parallel to the fault is permitted (for more details see Duesbery and Richardson (1991) and Vitek (1992)). We have, therefore, employed the constructed BOPs to calculate the (111) $\gamma$ surface using the simulation block consisting of 60 relaxed atoms. The result of this calculation is shown in figure 5 as a contour plot. 


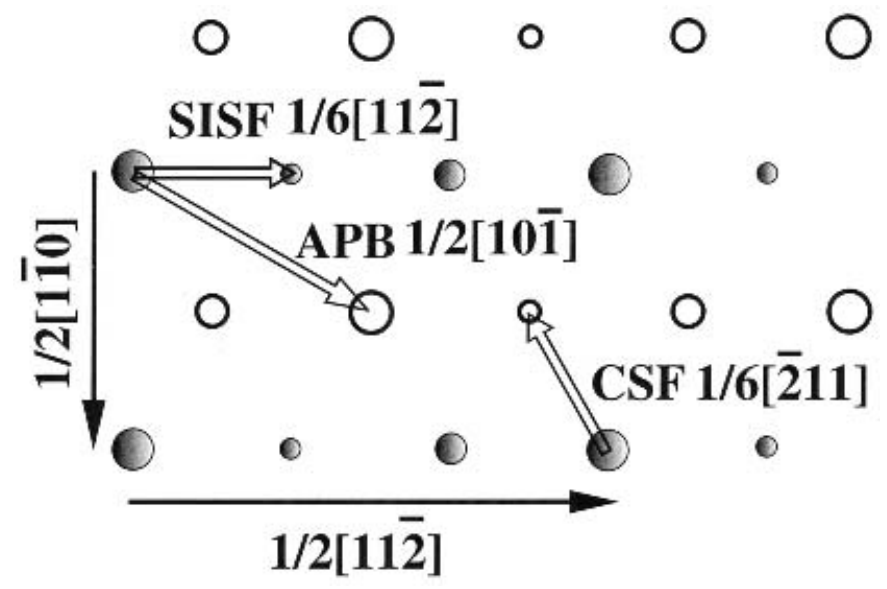

Figure 4. Three (111) planes of the $\mathrm{L1}_{0}$ structure. The atoms belonging to three adjacent atomic planes are distinguished by the size of the circles, the largest being from the top plane. The two different atomic species are depicted by open and full circles respectively. The three metastable stacking-fault-like defects, APB, CSF and SISF, are indicated by white arrows.

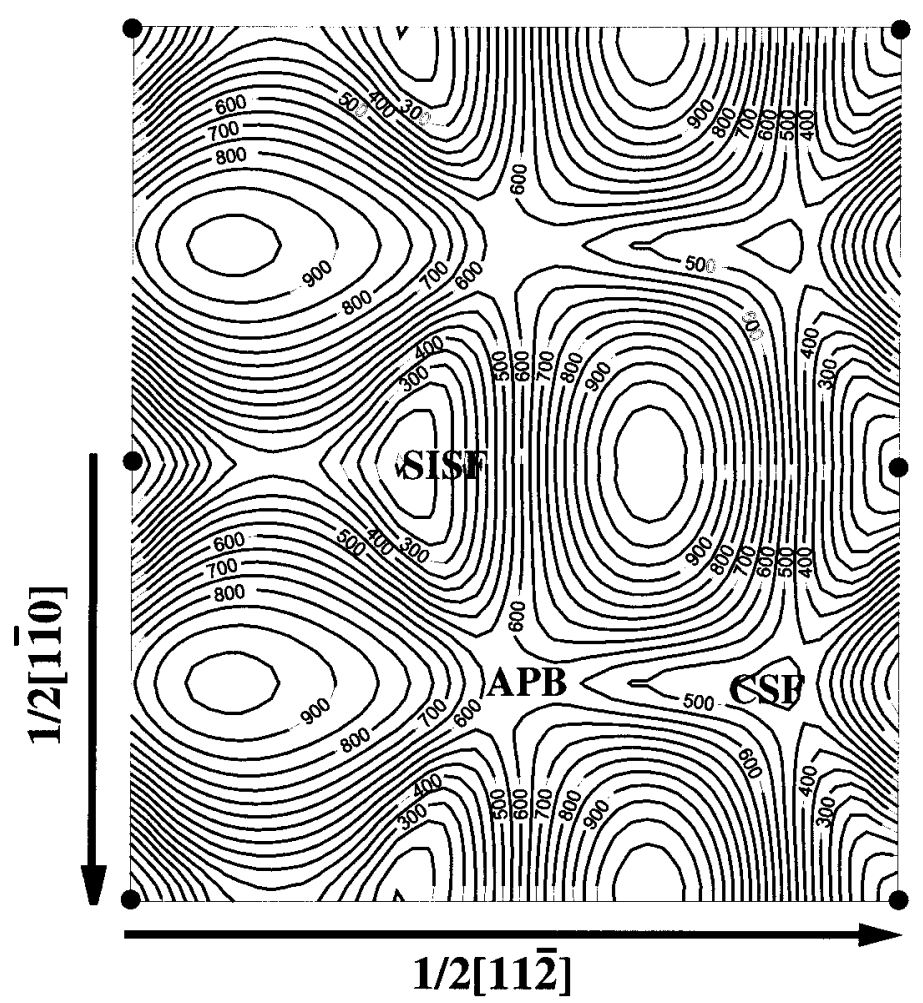

Figure 5. $\gamma$ surface for (111) plane in millijoules per square metre presented as a contour plot. Small full circles designate the points corresponding to the ideal crystal. 
The first important finding is that the $\gamma$ surface is everywhere positive so that shearing along the (111) plane does not lead to any instabilities with respect to the ideal lattice. The three minima seen on this surface correspond to the three stackingfault-type defects described below. The energies of these faults, calculated using BOPs, are compared in table 7 with those evaluated by two different ab-initio methods, FLAPW (Ehmann and Fähnle 1998) and FP LMTO (Vitek et al. 1997b) as well as with earlier studies that employed central-force many-body potentials of the FStype potential (Girshick and Vitek 1995, Vitek et al. 1997a) and the EAM (Rao et al. 1991, Simmons et al. 1993, Farkas 1994, Simmons et al. 1997, Panova and Farkas 1998). The agreement between the BOP and ab-initio values is reasonably good; in particular, the relatively high energy of the SISF is reproduced correctly. In contrast, the FS-type potential leads to too low a value of this energy. As already mentioned in the introduction, the first- and second-nearest-neighbour separations remain almost unchanged when creating this fault and for central forces the energy of the SISF is determined by the third and other more distant neighbours. The FS potential is very short range, cut off close to the third neighbours, the contribution of which is then very small. The EAM-type potentials are all cut off between fourth- and fifth-nearest neighbours so that contribution of the third (and possible fourth) neighbours may be significant. Thus potentials denoted P2 and P3, constructed by Simmons et al. (1993, 1997), lead to high values of the SISF energy but at the expense of unphysically strong interactions of third neighbours and associated poor reproduction of elastic constants. However, these potentials were developed with the aim of investigating the effect of the APB, CSF and SISF energies on the core structures of dislocations in the $\mathrm{L} 1_{0}$ structure rather than to obtain a realistic description of atomic interaction in TiAl. For the EAM-type potentials developed specifically for TiAl, namely those constructed by Farkas (1994) and the P1 potential constructed by Rao et al. (1991) (see also Simmons et al. $(1993,1997)$ ), the high value of the SISF energy predicted by ab-initio calculations cannot be obtained.

Table 7. Energies of stacking-fault like defects (APB, CSF and SISF) on the (111) plane and of the ordered twin calculated using the BOP with $c / a=1.016$ and interfacial energies of the $120^{\circ}$ rotational fault and pseudotwin calculated using the BOPs with $c / a=1.0$. The BOP predictions are compared with two different ab-initio calculations, calculations employing the FS-type potentials (Vitek et al. 1997a) and the EAM potentials. The latter are potentials constructed by Farkas (1994) (see also Panova and Farkas (1998)) and three different EAM-type potentials developed by Rao et al. (1991), denoted P1, P2 and P3 (see also Simmons et al. $(1993,1997)$ ). For more details, see text.

\begin{tabular}{lccccccc}
\hline & \multicolumn{7}{c}{ Energy $\left(\mathrm{mJ} \mathrm{m}^{-2}\right)$} \\
\cline { 2 - 8 } & APB & CSF & SISF & $\begin{array}{c}\text { Ordered } \\
\text { twin }\end{array}$ & $\begin{array}{c}120^{\circ} \\
\text { rotational fault }\end{array}$ & Pseudotwin & $\begin{array}{c}\text { Twin } \\
\text { with APB }\end{array}$ \\
\hline BOPs & 545 & 412 & 140 & 74.2 & 306 & 310 & 456 \\
FLAPW & 667 & 362 & 172 & 60 & 270 & 250 & 550 \\
FP LMTO & 710 & 314 & 134 & 109 & 235 & 213 & 501 \\
FS & 275 & 275 & 2.9 & 1.4 & 144 & 145 & 288 \\
EAM (Farkas 1994) & 322 & 308 & 60 & 40 & - & - & - \\
P1 & 306 & 119 & 71 & - & - & - & - \\
P2 & 550 & 320 & 220 & - & - & - & - \\
P3 & 770 & 580 & 250 & - & - & - & - \\
\hline
\end{tabular}


The relatively high energy of the SISF, obtained using the constructed shortrange $\mathrm{BOP}$, results principally from the change in the bond energy due to the angular dependence in the bond order (Pettifor et al. 1995). It should be noted that, while the metastability of the SISF is guaranteed by the symmetry of the $\mathrm{L1}_{0}$ structure, this is not the case for the APB and CSF (for example Paidar and Vitek 2002). Indeed, the ab-initio calculated $\gamma$ surface (Vitek et al. 1997b, Ehmann and Fähnle 1998) does not show a minimum corresponding to the APB but only an inflection. On the other hand, the $\gamma$ surface calculated using the present BOPs displays a shallow minimum corresponding to the APB. However, this subtle difference in the $\gamma$ surface is not significant when considering dislocation dissociation as explained further in $\S 5$.

\subsection{Energies of lamellar interfaces}

In lamellar TiAl-based alloys, whether in polycrystalline form or as polysynthetically twinned single crystals (Fujiwara et al. 1990), there are three variants of the twin-related $\gamma-\gamma$ interfaces (Feng et al. 1988, Schwartz and Sastry 1989, Inui et al. 1992a,b, Yamaguchi et al. 1996, 2000). They are

(i) ordered twins in which the [110] direction of the matrix is antiparallel to the [1110] direction of the twin,

(ii) $120^{\circ}$ rotational faults for which the [1 10$]$ direction of the matrix is parallel to

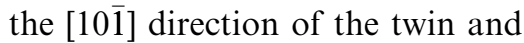

(iii) pseudotwins for which the [110] direction of the matrix is antiparallel to the $[10 \overline{1}]$ direction of the twin.

Furthermore, the ordered twin with additional APB was observed (Ricolleau et al. 1994) and studied in details employing high-resolution electron microscopy and the ab-initio FP LMTO method by Siegl et al. 1997. Using the constructed BOPs we calculated energies of these interfaces with fully relaxed atomic structures. The results are presented in table 7 where they are compared with two different ab-initio calculations as well as calculations employing FS-type potentials and for the ordered twin the EAM potential constructed by Farkas (1994). Because $c / a=1.016$ for the [101] vectors are 1.0085 times longer than the [110] TiAl and this results in a misfit at the $120^{\circ}$ rotational fault and the pseudotwin. In order to avoid this misfit, these two interfaces were modelled using the BOPs constructed for the ideal $c / a=1(\S 3.2)$; the ordered twin, both without and with an APB, was modelled with BOPs corresponding to $c / a=1.016$.

The agreement with the ab-initio calculations is very satisfactory. The case of the ordered twin again emphasizes the importance of directional bonding. The very low FS value, and relatively low value obtained within the EAM, have their roots, as in the case of the SISF, in the fact that the first- and second-nearest-neighbour separations are almost the same as in the ideal $\mathrm{L1}_{0}$ lattice. The present BOP predicts the ratios of the energies of the twin, the $120^{\circ}$ rotational fault and the pseudotwin to be $1: 4.1: 4.2$ which compares favourably with the results of ab initio calculations, particularly the FLAPW method that predicts $1: 4.5: 4.2$, as well as with the experimental values 1:5.8:6.7 deduced from atomic force microscopy (AFM) measurements ( $\mathrm{Lu}$ et al. 1996).

\subsection{Point defects}

Finally, we have calculated the energies associated with the formation of point defects in TiAl, including vacancies and antisites at both the Ti and the Al sites. It is 
now well recognized that the problem of calculating point defects in ordered alloys with two (or more) types of species is not trivial even for the simple stoichiometric case (Hagen and Finnis 1998). Here we have followed the statistical approach which introduces the concept of the 'raw' energy of the point defects (Woodward et al. 1998, Mishin and Herzig 2000). This is defined as the total change in the energy of the block used in the simulation when the defect (vacancy, antisite, etc.) was introduced and the block relaxed, while the number of lattice sites was kept fixed. These quantities are obtained directly from atomistic simulations and can be used to evaluate directly the energies of defects that do not change the stoichiometry of the TiAl compound. These are the exchange defects, $\mathrm{Al} \rightarrow \mathrm{Ti}$ and $\mathrm{Ti} \rightarrow \mathrm{Al}$, the double vacancy on $\mathrm{Ti}$ and $\mathrm{Al}$ sites, two Ti vacancies accompanied by the antisite $\mathrm{Ti} \rightarrow \mathrm{Al}$ and two $\mathrm{Al}$ vacancies accompanied by the antisite $\mathrm{Al} \rightarrow \mathrm{Ti}$. When $\epsilon_{\mathrm{coh}}$ denotes the cohesive energy per atom, $\epsilon_{\mathrm{V}_{\mathrm{Al}}}^{\text {raw }}$ and $\epsilon_{\mathrm{V}_{\mathrm{Ti}}}^{\text {raw }}$, the raw energies of the $\mathrm{Al}$ and Ti vacancies respectively, and $\epsilon_{\mathrm{Al} \rightarrow \mathrm{Ti}}^{\mathrm{raw}}$ and $\epsilon_{\mathrm{Ti} \rightarrow \mathrm{Al}}^{\mathrm{raw}}$ the raw energies of antisites respectively, the energies of these four defects that preserve the stoichiometry are (Woodward et al. 1998, Mishin and Herzig 2000)

exchange defect: $\epsilon_{\mathrm{Al} \rightarrow \mathrm{Ti}}^{\mathrm{raw}}+\epsilon_{\mathrm{Ti} \rightarrow \mathrm{Al}}^{\mathrm{raw}}$,

double vacancy: $2 \epsilon_{\mathrm{coh}}+\epsilon_{\mathrm{V}_{\mathrm{Al}}}^{\text {raw }}+\epsilon_{\mathrm{V}_{\mathrm{Ti}}}^{\text {raw }}$,

two vacancies and antisite: $2 \epsilon_{\mathrm{coh}}+2 \epsilon_{\mathrm{V}_{\mathrm{Ti}}}^{\mathrm{raw}}+\epsilon_{\mathrm{Ti} \rightarrow \mathrm{Al}}^{\mathrm{raw}}$,

two vacancies and antisite: $2 \epsilon_{\mathrm{coh}}+2 \epsilon_{\mathrm{V}_{\mathrm{Al}}}^{\mathrm{raw}}+\epsilon_{\mathrm{Al} \rightarrow \mathrm{Ti}}^{\text {raw }}$.

Furthermore, the effective formation energies $\epsilon^{\text {eff }}$, which depend on the concentrations of species forming the alloy, can be defined via statistical mechanics considerations so that the concentrations of the corresponding point defects are then proportional to $\exp \left(-\epsilon^{\mathrm{eff}} / k_{\mathrm{B}} \mathrm{T}\right)$ (Hagen and Finnis 1998, Woodward et al. 1998, Mishin and Herzig 2000). Following Mishin and Herzig (2000) the effective formation energies of vacancies at the $\mathrm{Al}$ and $\mathrm{Ti}$ sites in stoichiometric TiAl are

$$
\begin{aligned}
& \epsilon_{\mathrm{V}_{\mathrm{Al}}}^{\text {eff }}=\epsilon_{0}+\epsilon_{\mathrm{V}_{\mathrm{Al}}}^{\mathrm{raw}}+\frac{1}{4}\left(\epsilon_{\mathrm{Al} \rightarrow \mathrm{Ti}}^{\text {raw }}-\epsilon_{\mathrm{Ti} \rightarrow \mathrm{Al}}^{\mathrm{raw}}\right), \\
& \epsilon_{\mathrm{V}_{\mathrm{Ti}}}^{\text {eff }}=\epsilon_{0}+\epsilon_{\mathrm{V}_{\mathrm{Ti}}}^{\text {raw }}+\frac{1}{4}\left(\epsilon_{\mathrm{Ti} \rightarrow \mathrm{Al}}^{\mathrm{raw}}-\epsilon_{\mathrm{Al} \rightarrow \mathrm{Ti}}^{\mathrm{raw}}\right),
\end{aligned}
$$

and the effective antisite formation energies are

$$
\begin{aligned}
& \epsilon_{\mathrm{Ti} \rightarrow \mathrm{Al}}^{\text {eff }}=\frac{1}{2}\left(\epsilon_{\mathrm{Ti} \rightarrow \mathrm{Al}}^{\mathrm{raw}}+\epsilon_{\mathrm{Al} \rightarrow \mathrm{Ti}}^{\mathrm{raw}}\right), \\
& \epsilon_{\mathrm{Al} \rightarrow \mathrm{Ti}}^{\text {eff }}=\frac{1}{2}\left(\epsilon_{\mathrm{Al} \rightarrow \mathrm{Ti}}^{\mathrm{raw}}+\epsilon_{\mathrm{Ti} \rightarrow \mathrm{Al}}^{\mathrm{raw}}\right) .
\end{aligned}
$$

Table 8 compares the results of calculations of the energies of stoichiometry preserving defects and effective formation energies of vacancies and antisites cal-

Table 8. Effective formation energies of vacancies and antisites and energies of stoichiometry preserving defects calculated with BOPs for $\mathrm{TiAl}$, with the ab-initio method by Woodward et al. (1998) and by Mishin and Herzig (2000) using the EAM.

\begin{tabular}{lccccccc}
\hline & & & & \multicolumn{4}{c}{ Energy $(\mathrm{eV})$} \\
\cline { 6 - 8 } & $\begin{array}{c}\epsilon_{\mathrm{V}_{\mathrm{Ti}}}^{\text {eff }} \\
(\mathrm{eV})\end{array}$ & $\begin{array}{c}\epsilon_{\mathrm{V}_{\mathrm{Al}}}^{\text {eff }} \\
(\mathrm{eV})\end{array}$ & $\begin{array}{c}\epsilon_{\mathrm{Al} \rightarrow \mathrm{Ti}}^{\text {eff }} \\
(\mathrm{eV})\end{array}$ & $\begin{array}{c}\text { Exchange } \\
\text { defect }\end{array}$ & $\begin{array}{c}\text { Double } \\
\text { vacancy }\end{array}$ & $\begin{array}{c}\text { 2 Ti vacancies } \\
\text { + antisite }\end{array}$ & $\begin{array}{c}\text { 2 Al vacancies } \\
\text { + antisite }\end{array}$ \\
\hline BOPs & 1.05 & 1.28 & 0.82 & 1.65 & 2.33 & 2.92 & 3.38 \\
Ab-initio method & 1.59 & 1.99 & 0.60 & 1.20 & 3.58 & 3.78 & 4.59 \\
EAM & 1.15 & 1.41 & 0.44 & 0.88 & 2.56 & 2.74 & 3.25 \\
\hline
\end{tabular}


culated using the constructed BOPs for TiAl with the calculations of Woodward (1998) who employed an ab-initio method and those of Mishin and Herzig (2000) who used the EAM. In all these calculations the atomic positions were fully relaxed. As can be seen from this table, all the calculations show the same ordering of the energies. However, both the BOP and the EAM give generally lower values of the energies than those found in the ab-initio calculations, which may be related to the completeness of atomic relaxations.

\section{§. CONCLUSIONS}

(i) We have presented a general formalism for developing BOPs for binary intermetallic compounds that consist of transition and sp-valent elements. In this paper we applied this formalism to TiAl with the tetragonal $\mathrm{Ll}_{0}$ structure. The most important new feature of the present scheme is that the binding energy is composed not only of the attractive bond part and the repulsive term described by a pair potential but also an additional environmentally dependent contribution reflecting the strong core repulsion by the valence sp electrons in transition metals and their alloys. This term, represented by a many-body central-force potential of Yukawa type, allows us to reproduce the negative Cauchy pressures observed in many intermetallic compounds including TiAl. For the bond contribution, we have used the TB pd approximation in which $\mathrm{d}-\mathrm{d}$, $\mathrm{p}-\mathrm{p}$ and $\mathrm{d}-\mathrm{p}$ bond integrals have been deduced from the first-principles non-canonical screened LMTO method. However, no explicit environmentally dependent screening of the bond integrals is needed in TiAl owing to their short-range nature in closepacked structures.

(ii) The fitting procedure is sequential, fitting first the bond part, using only the data obtained from DFT-based ab-initio calculations, followed by construction of the environmentally dependent repulsive term so as to reproduce Cauchy pressures, and finally determining parameters of the pair potential by fitting the cohesive energies, the equilibrium lattice parameters and remaining elastic constants. Each of these contributions can be separated into three parts that correspond to $\mathrm{Ti}-\mathrm{Ti}, \mathrm{Al}-\mathrm{Al}$ and $\mathrm{Ti}-\mathrm{Al}$ interactions. However, no physical property of pure Al in the fcc ground state was fitted when constructing the potentials and thus the AlAl interaction should not be used to represent a BOP for elemental aluminium. In the case of $\mathrm{Ti}-\mathrm{Ti}$ the original fitting was to properties of hexagonal Ti but these interactions were slightly modified when determining $\mathrm{Ti}-\mathrm{Al}$ interactions, as described in $\S 3$. Hence, $\mathrm{Ti}-\mathrm{Ti}$ interactions employed for $\mathrm{TiAl}$, the parameters of which are summarized in tables 1 and 2 and in the text, should also not be used to represent the BOP for elemental Ti. The BOP for pure $\mathrm{Ti}$, which includes the environmentally dependent contribution to the binding energy, will be published elsewhere.

(iii) A remarkable property of the BOPs constructed for TiAl is their excellent transferability to $3: 1$ stoichiometry of $\mathrm{Ti}_{3} \mathrm{Al}$. The potentials predict correctly that the most stable structure is hexagonal $\mathrm{D} 0_{19}$, give cohesive energies and lattice parameters very close to the experimental values and predict an elastic constant that compare reasonably well with those 
calculated by the ab-initio method. This is principally due to the transferability of the screened bond integrals within close-packed structures. Thus the constructed BOPs can be used not only for $1: 1$ stoichiometry of TiAl but for stoichiometries corresponding to different higher contents of $\mathrm{Ti}$, albeit not for pure Ti. Furthermore, the potentials predict the relative stability order of different structures at the same composition as for TiAl from $\mathrm{L} 1_{0} \rightarrow \mathrm{B} 19 \rightarrow \mathrm{B} 2 \rightarrow \mathrm{B} 1$, in agreement with ab-initio calculations.

(iv) The mechanical stability of the $\mathrm{L}_{0}$ structure when employing the constructed BOPs has been tested by applying homogeneous expansions and contractions and by studying three distinct transformation paths. These calculations demonstrate that the $\mathrm{L} 1_{0}$ structure is stable with respect to large deformations.

(v) The most important test related to the plastic properties of $\mathrm{L}_{0}$ TiAl is the calculation of the $\gamma$ surface for the $\{111\}$ planes which are the slip and twinning planes. This calculation demonstrates that shearing along these planes does not lead to any instabilities and suggest metastability of three commonly assumed stacking-fault-like defects, APB, CSF and SISF, on these planes. The calculated energies of these faults are in a very good agreement with those evaluated by ab-initio methods. This is particularly remarkable in the case of the SISF, the high energy of which arises owing to the angular dependence of atomic interactions that is correctly accounted for in the present scheme. However, a disparity between $a b$ initio calculations and calculations using BOP occurs concerning the stability of the APB. The latter suggest that the APB is not metastable since only an inflection rather than a minimum on the $\gamma$ surface is found for the displacement corresponding to the APB (Vitek et al. 1997b, Ehmann and Fähnle 1998). The APB may play a role when considering

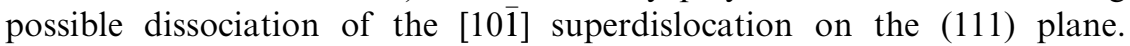
When this fault is stable, this superdislocation may dissociate into two superpartials with the Burgers vectors $\frac{1}{2}[10 \overline{1}]$ joined by the APB. On the other hand, if the APB is unstable and the $\gamma$ surface possesses only an inflection a core spreading with two distinct peaks, separated by two or three lattice spacings, can be expected (Vitek et al. 1972, Vitek 1992, Duesbery et al. 2002). Yet, since the energy of the metastable APB found using the BOP is very high, the separation of the superpartials will be only a few lattice spacings and thus the two cases will be almost indistinguishable. However, an alternative is that, when the APB energy is very high and/or the APB is not metastable, the dissociation of the $[10 \overline{1}]$ superdislocation will involve the SISF and CSF that possess lower energies. This has, indeed, been found in the preliminary atomistic studies of the core of the superdislocation (Znam 2001).

(vi) Finally, three distinct $\gamma-\gamma$ interfaces found in the lamellar TiAl and point defects composed of vacancies and antisites were studied using the constructed BOPs. In the former case the ratios of energies of the ordered twin, $120^{\circ}$ rotational fault and pseudotwin are in good agreement with ab-initio data and with measurements using AFM. In the latter case the agreement with available ab-initio calculations is only qualitative in that the orders of energies of different point defects are the same in both cases. 


\section{ACKNOWLEDGEMENTS}

This research was supported in part by the National Science Foundation grant DMR99-81023 (S.Z. and V.V.), US Department of Energy, Basic Energy Sciences grant DE-FG02-98ER45702 (S.Z., V.V. and D.N.M.) and by Sanyo Electric Co. (D.N.M. and D.G.P.). The computations have been performed in part at the Materials Modelling Laboratory, Department of Materials, University of Oxford, on the Hewlett-Packard Exemplar computer which was jointly funded by HewlettPackard and Higher Education Funding Council of England under the Joint Equipment Research Initiative scheme. The authors acknowledge stimulating discussions with O. K. Andersen, M. Aoki, D. Dimiduk, M. Fähnle, H. Inui, Y. W. Kim, M. Mrovec, H. Nakamura, Y. Mishin, V. Paidar, M. Sob, K. Tanaka, P. Veyssière, C. Woodward and M. Yamaguchi. We also thank R. Porizek for his help in preparing this paper.

\section{REFERENCES}

Ackland, G. J., Tichy, G., Vitek, V., and Finnis, M. W., 1987, Phil. Mag. A, 56, 735. Andersen, O. K., and Jepsen, O., 1984, Phys. Rev. Lett., 53, 2571.

Andersen, O. K., JePSEn, O., and GlötZel, D., 1985, Highlight of Condensed Matter Theory, edited by F. Bassani, F. Fumi and M. P. Tosi (Amsterdam: North-Holland), p. 59.

Aoki, M., 1993, Phys. Rev. Lett., 71, 3842.

Aoki, M., and Pettifor, D. G., 1993, Mater. Sci. Engng, A176, 19; 1994, Physics of Transition Metals, edited by P. M. Oppeneer and J. Kubler (Singapore: World Scientific), p. 299.

Appel, F., and Wagner, R., 1998, Mater. Sci. Engng, R22, 187.

Cahn, R. W., 1995, Phil. Trans. R. Soc. A, 351, 497.

Carlsson, A. E., 1991, Phys. Rev. B, 44, 6590.

CASTEP 3.9, (Academic version) 1999, Rev. mod. Phys., 64, 1045.

Cottrell, A., 1998, Concepts in the Electron Theory of Alloys (London: Institute of Materials), p. 124.

Daw, M. S., and Baskes, M. I., 1984, Phys. Rev. B, 29, 6443.

Dimiduk, D. M., 1998, Intermetallics, 6, 613; 1999, Mater. Sci. Engng, A263, 281.

Duesbery, M. S., and Richardson, G. Y., 1991, CRC Crit. Rev. Solid St. Mater. Sci., 17, 1.

Duesbery, M. S., Vitek, V., and Cresti, J., 2002, Understanding Materials: Professor Sir Peter Hirsch Festschrift, edited by C. J. Humphreys (London: Institute of Materials), p. 165.

Ehmann, J., and Fähnle, M., 1998, Phil. Mag. A, 77, 701.

FARKaS, D., 1994, Modelling Simulation Mater. Sci. Engng, $2,975$.

Feng, C. R., Mitchel, D. J., and Crowe, C. R., 1988, Scripta metall., 22, 1481.

Finnis, M., and Sinclair, J. E., 1984, Phil. Mag. A, 50, 45.

Folles, S. M., 1993, Phys. Rev. B, 48, 4287.

Froes, F. H., and Suryanarayama, C., 1996, Physical Metallurgy and Processing of Intermetallic Compounds, edited by N. S. Stoloff and V. K. Sikka (London: Chapman \& Hall), p. 297.

Fu, C. L., and Yoo, M. H., 1990, Phil. Mag. Lett., 62, 159; 1992, Mater. chem. Phys., 32, 25.

Fu, C. L., Zou, J., and Yoo, M. H., 1995, Scripta. metall. mater., 33, 885.

Fujiwara, T., Nakamura, A., Hosomi, M., Nishitani, S. R., Shirai, Y., and Yamaguchi, M., 1990, Phil. Mag. A, 61, 591.

Girshick, A., Bratkovsky, A. M., Pettifor, D. G., and Vitek, V., 1998, Phil. Mag. A, 77, 981.

Girshick, A., and Vitek, V., 1995, High-Temperature Ordered Intermetallic Alloys VI, Materials Research Society Symposium Proceedings, Vol. 364, edited by J. Horton, I. Baker, S. Handada, R. D. Noebe and D. Schwartz (Pittsburgh, Pennsylvania: Materials Research Society), p. 145.

Haas, H., Wang, C. Z., Fähnle, M., Elsässer, C., and Ho, K. M., 1998, Phys. Rev. B, 58, 1461. 
Hagen, M., and Finnis, M., 1998, Phil. Mag. A, 77, 447.

Horsfield, A. P., and Bratkovsky, A. M., 1996, Phys. Rev. B, 53, 15381.

Horsfield, A. P., Bratkovsky, A. M., Fearn, M., Pettifor, D. G., and Aoki, M., 1996a, Phys. Rev. B, 53, 12694.

Horsfield, A. P., Bratkovsky, A. M., Pettifor, D. G., and Aoki, M., 1996b, Phys. Rev. $\mathrm{B}, \mathbf{5 3}, 1656$.

Hultgren, R., Orr, R. L., Anderson, P. D., and Kelley, K. K., 1973, Selected Values of the Thermodynamic Properties of Metals and Binary Alloys (New York: Wiley).

Inui, H., Kishida, K., Misaki, M., Kobayashi, M., Shirai, Y., and Yamaguchi, M., 1995, Phil. Mag. A, 72, 1609.

Inui, H., Matsumuro, M., Wu, D. H., and Yamaguchi, M., 1997, Phil. Mag. A, 75, 395.

Inui, H., Nakamura, A., Oh, M. H., and Yamaguchi, M., 1992a, Phil. Mag. A, 66, 557.

Inui, H., Oh, M. H., Nakamura, A., and Yamaguchi, M., 1992b, Acta metall. mater., 40, 3095.

Kad, B. K., and Hazzledine, P. M., 1992, Phil. Mag. Lett., 66, 133.

Kawabata, T., Kanai, T., and Izumi, O., 1985, Acta metall., 33, 1355; 1991, Phil. Mag. A, 63, 1291.

Kim, M., Nomura, M. Vitek, V., and Pope, D., 1999, High-Temperature Ordered Intermetallic Alloys VIII, Materials Research Society Symposium Proceedings, Vol. 552, edited by E. Gorge, M. Mills and M. Yamaguchi (Pittsburgh, Pennsylvania: Materials Research Society), p. KK3.1.1.

KIM, Y. W., 1994, J. Metals, 46, 30; 1998, Intermetallics, 6, 623.

Kishida, K., Inui, H., and Yamaguchi, M., 1998, Phil. Mag. A, 78, 1; 1999, Intermetallics, 7, 1131 .

Loria, E. A., 2000, Intermetallics, 8, 1339; 2001, ibid., 9, 997.

Lu, L., Siegl, R., Girshick, A., Pope, D. P., and Vitek, V., 1996, Scripta mater., 34, 971.

McCullough, C., Valencia, J. J., Mateous, H., Levi, C. G., Mehrabian, R., and RhYNe, K. A., 1988, Scripta metall., 22, 1131.

Mishin, V., and Herzig, C., 2000, Acta mater., 48, 589.

Moriarty, J. A., 1990, Phys. Rev. B, 42, 1609.

Moriarty, J. A., and Widom, M., 1997, Phys. Rev. B, 56, 7905.

NaKamura, H., 1999, D. Phil. Thesis, University of Oxford, Oxford, UK.

Nakamura, H., Nguyen-Mahn, D., and Pettifor, D. G., 2000, J. Alloys Compounds, 306, 113.

Nguyen-Manh, D., Bratkovsky, A. M., and Pettifor, D. G., 1995, Phil. Trans. R. Soc. A, 351, 529.

Nguyen-Manh, D., and Pettifor, D. G., 1999a, Gamma Titanium Allumides, edited by Y. W. Kim (Warrendale, Pennsylvania: Metallurgical Society of AIME), p. 175; 1999b, Intermetallics, 7, 1005.

Nguyen-Manh, D., Pettifor, D. G., Shao, G., Miodownik, A. P., and Pasturel, A., 1996, Phil. Mag. A, 74, 1385.

Nguyen-Manh, D., Pettifor, D. G., and Vitek, V., 2000, Phys. Rev. Lett., 85, 4136.

Nguyen-Manh, D., Pettifor, D. G., Znam, S., and Vitek, V., 1998, Tight-Binding Approach to Computational Materials Science, Materials Research Society Symposium Proceedings, Vol. 491, edited by P. E. A. Turchi, A. Gonis and L. Colombo (Pittsburgh, Pennsylvania: Materials Research Society), p. 353.

Paidar, V., and Vitek, V., 2002, Intermetallic Compounds: Principles and Practice, Vol. III, edited by J. H. Westbrook and R. L. Fleisher (New York: Wiley), p. 437.

Paidar, V., Wang, L. G., Sob, M., and Vitek, V., 1999, Modeling Simulation Mater. Sci. Engng, 7, 369.

Panova, J., and Farkas, D., 1998, Phil. Mag. A, 78, 389.

Pearson, W. B., 1967, A Handbook of Lattice Spacings and Structures of Metals and Alloys (Oxford: Pergamon).

Pettifor, D. G., 1978, J. Phys. F, 8, 219; 1989, Phys. Rev. Lett., 63, 2480; 1995, Bonding and Structures of Molecules and Solids (Oxford University Press).

Pettifor, D. G., Aoki, M., Gumbsch, P., Horsfield, A. P., Nguyen-Manh, D., and Vitek, V., 1995, Mater. Sci. Engng, A192-A193, 24.

Pettifor, D. G., and Podloucky, R., 1984, Phys. Rev. Lett., 53, 1080. 
Pettifor, D. G., and Ward, M. A., 1984, Solid St. Commun., 49, 291.

Pugh, S. F., 1954, Phil. Mag., 45, 823.

Rao, S. I., Woodward, C., and Parthasarathy, T. A., 1991, High-Temperature Ordered Intermetallic Alloys IV, Materials Research Society Symposium Proceedings, Vol. 213, Edited by L. A. Johnson, D. P. Pope and J. O. Stiegler (Pittsburgh, Pennsylvania: Materials Research Society), p. 125.

Ricolleau, C., Denquin, A., and Naka, S., 1994, Phil. Mag. Lett., 69, 197.

Schwartz, D. S., and Sastry, S. M. L., 1989, Scripta metall., 23, 1621.

Siegl, R., Vitek, V., Inui, H., Kishida, K., and Yamaguchi, M., 1997, Phil. Mag. A, 75, 1447.

Simmons, J. P., Rao, S. I., and Dimiduk, D., 1993, High-Temperature Ordered Intermetallic Alloys, Materials Research Society Symposium Proceedings, Vol. 288, edited by I. Baker, R. Darolia, J. D. Whittenberger and M. H. Yoo (Pittsburgh, Pennsylvania: Materials Research Society), p. 335; 1997, Phil. Mag. A, 75, 1299.

Skinner, A. J., and Pettifor, D. G., 1991, J. Phys.: condens. Matter, 3, 2029.

Soв, M., and Viтeк, V., 1996, Stability of Materials, NATO Advanced Study Institute Series, edited by A. Gonis, P. E. A. Turchi and J. Kudrnovsky (New York: Plenum), p. 449.

Sob, M., Wang, L. G., and Vitek, V., 1997, Comput. Mater. Sci., 8, 100.

Song, Y., Tang, S. P., Xu, J. H., Mryasov, O. N., Freeman, A. J., Woodward, C., and Dimiduk, D. M., 1994, Phil. Mag. B, 70, 987.

Stoloff, N. S., Liu, C. T., and Deevi, S. C., 2000, Intermetallics, 8, 1313.

Sutton, A., Finnis, M. W., Pettifor, D. G., and Ohta, Y., 1988, J. Phys. C, 21, 35.

Tanaka, K., Ichitsubo, T., InUi, H., Yamaguchi, M., and Koiwa, M., 1996a, Phil. Mag. Lett., 73, 71.

Tanaka, K., and Koiwa, M., 1996, Intermetallics, 4, S29.

Tanaka, K., Okamoto, K., Inui, H., Minonishi, Y., Yamaguchi, M., and Koiwa, M., 1996b, Phil. Mag. A, 73, 1475.

Tetsui, K., 1999, Curr. Opin. Solid St. Mater. Sci., 4, 243.

van Schilfgatrde, M., Paxton, A. T., Pasturel, A., and Methfessel, M., 1991, Alloys Phase Stability and Design, Materials Reserch Society Symposium Proceedings, Vol. 186, edited by G. M. Stocks, D. P. Pope and F. Giamei (Pittsburgh, Pennsylvania: Materials Research Society), p. 107.

Vitek, V., 1968, Phil. Mag. A, 18, 773; 1992, Prog. Mater. Sci., 36, 1.

Vitek, V., Girshick, A., Siegl, R., Inui, H., and Yamaguchi, M., 1997a, Properties of Complex Inorganic Solids, edited by A. Gonis, P. E. A. Turchi, and A. Meike (New York: Plenum), p. 355.

Vitek, V., Ito, K., Siegl, R., and Znam, S., 1997b, Mater. Sci. Engng, A240, 752.

Vitek, V., LejCeK, L., and Bowen, D. K., 1972, Interatomic Potentials and Simulations of Lattice Defects, edited by P. C. Gehlen, J. R. Beeler and R. I. Jaffee (New York: Plenum), p. 493.

Westbrook, J. H., 1996, Mater. Res. Soc. Bull., 21, 37.

Westbrook, J. H., and Fleisher, R. L. (editors), 2002, Intermetallic Compounds: Principles and Practice (New York: Wiley).

Widom, M., Allehyani, I., and Moriarty, J. A., 2000, Phys. Rev. B, 62, 3648.

Woodward, C., Kajihara, S., and Yang, L. H., 1998, Phys. Rev. B, 57, 13459.

Woodward, C., and Maclaren, J. M., 1996, Phil. Mag. A, 74, 337.

Woodward, C., Maclaren, J. M., and Rao, S., 1992, J. Mater. Res., 7, 1735.

Yamaguchi, M., Inui, H., and Ito, K., 2000, Acta mater., 48, 307.

Yamaguchi, M., Inui, H., Yokoshima, S., Kishida, K., and Johnson, D. R., 1996, Mater. Sci. Engng, A213, 25.

Zhang, F., Chen, S. L., Chang, Y. A., and Kattner, U. R., 1997, Intermetallics, 5, 471.

Znam, S., 2001, PhD Thesis, University of Pennsylvania, Philadelphia, Pennsylvania, USA.

Znam, S., Nguyen-Manh, D., Pettifor, D. G., and Vitek, V., 2000, Properties of Complex Inorganic Solids 2, edited by A. Meike, A. Gonis and P. E. A. Turchi (Dordrecht: Kluwer-Plenum), p. 479.

Zou, J., Fu, C. L., and Yoo, M. H., 1995, Intermetallics, 3, 265. 